\title{
The Logic of Brouwer and Heyting
}

\author{
Joan Rand Moschovakis
}

November 30, 2007

Intuitionistic logic consists of the principles of reasoning which were used informally by L. E. J. Brouwer, formalized by A. Heyting (also partially by V. Glivenko), interpreted by A. Kolmogorov, and studied by G. Gentzen and K. Gödel during the first third of the twentieth century. Formally, intuitionistic first-order predicate logic is a proper subsystem of classical logic, obtained by replacing the law of excluded middle $\mathrm{A} \vee \neg \mathrm{A}$ by $\neg \mathrm{A} \supset(\mathrm{A} \supset \mathrm{B})$; it has infinitely many distinct consistent axiomatic extensions, each necessarily contained in classical logic (which is axiomatically complete). However, intuitionistic second-order logic is inconsistent with classical second-order logic. In terms of expressibility, the intuitionistic logic and language are richer than the classical; as Gödel and Gentzen showed, classical first-order arithmetic can be faithfully translated into the negative fragment of intuitionistic arithmetic, establishing proof-theoretical equivalence and clarifying the distinction between the classical and constructive consequences of mathematical axioms.

The logic of Brouwer and Heyting is effective. The conclusion of an intuitionistic derivation holds with the same degree of constructivity as the premises. Any proof of a disjunction of two statements can be effectively transformed into a proof of one of the disjuncts, while any proof of an existential statement contains an effective prescription for finding a witness. The negation of a statement is interpreted as asserting that the statement is not merely false but absurd, i.e., leads to a contradiction. Brouwer objected to the general law of excluded middle as claiming a priori that every mathematical problem has a solution, and to the general law $\neg \neg \mathrm{A} \supset \mathrm{A}$ of double negation as asserting that every consistent mathematical statement holds.

Brouwer called "the First Act of Intuitionism" the separation of mathematics (an exclusively mental activity) from language. For him, all mathematical objects (including proofs) were mental constructions carried out by individual mathematicians. This insight together with "the intuition of the bare two-oneness" guided the creation of intuitionistic logic and arithmetic, with full induction and a theory of the rational numbers. It was limited, by Brouwer's early insistence on predicative definability, to the construction of entities which were finitely specifiable and thus at most denumerably infinite in number; this seemed to exclude the possibility of a good intuitionistic theory of the continuum.

A later insight led Brouwer to "the Second Act of Intuitionism," which accepted infinitely proceeding sequences of independent choices of objects already constructed (choice sequences of natural numbers, for example) as legitimate mathematical entities. He succeeded in extracting positive information about the structure of the continuum from the fact that an individual choice sequence may be known only by its finite approximations. His "Bar Theorem" (actually a new mathematical axiom) essentially accepted induction up to any denumerable ordinal, significantly extending the intuitionistically available part of classical arithmetic and analysis. His Continuity Principle, which contradicted classical logic (but not classical first-order arithmetic), made possible the development of a rich al- 
ternative theory of the continuum. Brouwer's constructive understanding of the universal and existential quantifiers led him to accept some choice principles stronger than those of classical mathematics, and at the same time to reject the full classical axiom of choice.

Because intuitionistic logic is an ex post facto construction reflecting the forms of reasoning actually used in intuitionistic mathematics, in order to give a full account of the logic we will need to consider some of the mathematics as well. And because "the possibilities of thinking cannot be reduced to a finite number of rules constructed in advance," we would do well to remember that this is not the last word in the subject.

\section{Historical Development of Intuitionistic Logic}

The Dutch mathematician and logician Luitzen Egbertus Jan Brouwer was born February 27, 1881 in Overschie, near Rotterdam, and died December 2, 1966 in Blaricum, east of Amsterdam. He was a highly original thinker who developed an intuitionistic alternative to classical mathematics, introduced the term "metamathematics" for the mathematical study of logical reasoning, proved fundamental theorems on continuous groups and on the fixed points of continuous mappings, and invented many of the basic notions of algebraic topology in the process of establishing the topological invariance of dimension. ${ }^{2}$

Brouwer earned a master's degree from the University of Amsterdam in 1904 with a thesis on continuous motions in real four-dimensional space. His doctoral dissertation [11], written under the delicate supervision of D. J. Korteweg and presented in 1907, was titled "On the Foundations of Mathematics" (Over de grondslagen der wiskunde) and divided into three parts: "The construction of mathematics," "Mathematics and experience" and "Mathematics and logic." Korteweg refused to accept some controversial material, but his student's critiques of the best-known mathematical philosophies of the time remained.

In Part I Brouwer built up the positive and negative integers and their arithmetic, then the rationals with their denumerable dense ordering, and finally a "measurable continuum," the result of constructing a scale of order type $\eta$ (the order type of the rationals) on the raw one-dimensional continuum given by intuition. ${ }^{3}$ In Part II he described the origin of scientific activity in the observation of "causal systems in time," disagreed with Kant by asserting that time is the only a priori element in science, and distinguished between mathematics ("an action and not a science") and metamathematics ("an application of mathematics, that is ... an experimental science"). ${ }^{4,5}$

Part III began with the argument that mathematics is independent of and prior to logic, which depends on it; this was a direct and immediate challenge to the logicism of Frege and Russell, and also to the formalism of David Hilbert who was then the most influential logician (possibly the most influential mathematician) in Europe. Brouwer went on to criticize Cantor's set theory, accepting in principle every countable ordinal but rejecting

\footnotetext{
${ }^{1}$ A. Heyting, from the introduction to [56]; the translation is by A. S. Troelstra [116].

${ }^{2}$ In one dimension, Brouwer's fixed point theorem implies that every continuous mapping of the real unit interval $[0,1]$ into itself holds at least one point fixed. The invariance of dimension means that if there is a one-to-one continuous mapping from real $n$-space into real $m$-space, then $n \leq m$.

${ }^{3} \mathrm{~A}$ linear ordering is of type $\eta$ if and only if it is denumerable, has neither a smallest nor a largest element, and has the property that between any two elements there is another element.

${ }^{4}$ Arguing against Russell's defense of the Kantian a priority of space, Brouwer asserted that threedimensional Euclidean geometry is just a six-parameter group which has proved useful to man in understanding and controlling the "phenomena of his world of experience."

${ }^{5}$ Hilbert adopted the name "metamathematics" for his program of attempting to establish, by finitary means, the consistency of formal systems representing logical and mathematical reasoning.
} 
Cantor's second number class (the class of all countable ordinals) as a completed totality because, being nondenumerable, it "cannot be mathematically constructed." ${ }^{6}$ Finally, he argued in detail against logicism (which he attributed to Russell and Peano) and formalism.

The summary contained the following main points:

- "Mathematics is created by a free action independent of experience; it develops from a single aprioristic basic intuition, which may be called invariance in change as well as unity in multitude." 7

- "Further, projecting mathematical systems on experience is also a free action, which shows itself efficient in the struggle for life; ..."

- "A logical construction of mathematics, independent of the mathematical intuition, is impossible - for by this method no more is obtained than a linguistic structure, which irrevocably remains separated from mathematics..."

In April 1908 Brouwer had the honor of addressing the International Congress of Mathematicians in Rome, on the theory of Lie groups. The French preintuitionist Poincare also spoke at the conference, on the future of mathematics. ${ }^{8}$ In Part III of his doctoral dissertation Brouwer had already analyzed Poincare's objections to logicism and to Cantor's set theory: "He blames mainly in logistics the petitio principii and in Cantorism the hypothesis of the actual infinite. But hereby he does not touch upon the heart of the question, which is situated deeper, namely in the confusion between the act of constructing mathematics and the language of mathematics."

That same year, in "The unreliability of the logical principles" (De onbetrouwbaarheid der logische principes), Brouwer asked,

Is it allowed, in purely mathematical constructions and transformations, to neglect for some time the idea of the mathematical system under construction and to operate in the corresponding linguistic structure, following the principles of syllogism, of contradiction and of tertium exclusum, and can we then have confidence that each part of the argument can be justified by recalling to the mind the corresponding mathematical construction?

His answer was to argue for modus ponens (syllogism) and the law of contradiction but against the universal validity of the principle of excluded middle for properties of infinite

\footnotetext{
${ }^{6}$ Here Brouwer introduced the notion of a denumerably unfinished set, with "the following properties: we can never construct in a well-defined way more than a denumerable subset of it, but when we have constructed such a subset, we can immediately deduce from it, following some previously defined mathematical process, new elements which are counted to the original set. But from a strictly mathematical point of view this set does not exist as a whole; nor does its power exist; however we can introduce these words here as an expression for a known intention." Thus he rejected Cantor's platonism.

${ }^{7}$ Brouwer's footnote to the first point read: "The first act of construction has two discrete things thought together (also according to Cantor, Vortrag auf der Naturforscherversammlung in Kassel 1903); F. Meyer (Verhandl. internat. Math. Congr. Heidelberg 1904, p. 678) says that one thing is sufficient, because the circumstance that I think of it can be added as a second thing; this is false, for exactly this adding (i.e., setting it while the former is retained) presupposes the intuition of two-ity; only afterwards this simplest mathematical system is projected on the first thing and the ego which thinks the thing."

Meyer's claim, to which Brouwer objected, brings to mind the beginning of Dedekind's argument (p. 64 in the 1901 English translation of [35]) for the existence of infinite sets: "My own realm of thoughts, i.e., the totality $S$ of all things, which can be objects of my thought, is infinite. For if $s$ signifies an element of $S$, then is the thought $s^{\prime}$, that $s$ can be object of my thought, itself an element of $S . " \quad$ In the preface Dedekind asserted that "numbers are free creations of the human mind."

${ }^{8}$ Dale M. Johnson, "Brouwer's coming of age as a topologist," page 65.
} 
systems, establishing a basis for what is now known as intuitionistic propositional logic. Observing that the law of contradiction justifies the double negation of the law of excluded middle, he added in a footnote: "Consequently, the theorems which are usually considered as proved in mathematics, ought to be divided into those that are true and those that are non-contradictory."

Following Korteweg's advice, Brouwer spent the next few years solving fundamental problems in topology. His inaugural lecture as an unpaid privatdocent or lecturer, given in 1909, was on the foundations of geometry and topology and included his view that "the intuition of time or intuition of two-in-one" lies behind all mathematical endeavor; while the nineteenth-century discovery of projective and other non-Euclidean geometries refuted Kant's belief in the a priority of (Euclidean) space. ${ }^{9}$ His "On analysis situs," with ingenious topological counterexamples, and two papers on perfect sets quickly followed.

In 1912 these efforts were rewarded with a permanent appointment as Professor of Mathematics at the University of Amsterdam. An English translation of Brouwer's inaugural lecture, "Intuitionism and formalism," appeared in 1913 in the Bulletin of the American Mathematical Society. Here Brouwer used the term "neo-intuitionism" to refer to his own philosophy, distinguishing it from the old-style intuitionism which accepted Kant's belief in the a priority of both space and time. He objected strongly to the formalist position, which he identified with the belief that a mathematical theory can be justified by separating its content from its form (as in Peano's formal system for first-order arithmetic), and then proving the impossibility of deriving both a sentence (thought of as a string of symbols) and its negation from the axioms of the theory using formal logic.

The professorship gave him the freedom to continue developing and refining intuitionistic mathematics and mathematical philosophy, most remarkably in [14] and [15], as well as the stature to profoundly influence the ongoing foundational discussion which had begun before the turn of the century and was still going strong in 1930. It also brought him students, of whom Arend Heyting (1898 - 1980) was the one most devoted to intuitionism. Apparently realizing the desirability of communicating his own ideas in a way that would be comprehensible to the general mathematical community, Brouwer suggested that Heyting take as his doctoral subject the axiomatization and formalization of intuitionistic logic and parts of mathematics. Heyting responded with enthusiasm, and in 1925 he defended his dissertation, on the axiomatization of intuitionistic projective geometry. Related articles appeared in the Mathematische Annalen in $1927 .{ }^{10}$

In 1923 ([16], in Dutch) Brouwer published "Intuitionist splitting of the fundamental notions of mathematics," stressing the distinction between the classical meaning of negation as falsity and Brouwer's stronger interpretation of negation as absurdity. A German version [20] and addendum [23] followed. W. P. van Stigt points out ([87], p. 275) that this paper marked a radical departure for Brouwer, as it contained the statement and proof of a purely logical theorem: "Absurdity-of-absurdity-of-absurdity is equivalent with absurdity." It also demonstrated the use of negation and double negation as tools for defining new relations between mathematical objects; for example, beginning with the constructively defined relations of coincidence and apartness between points of the plane, Brouwer

\footnotetext{
${ }^{9}$ In the same lecture Brouwer traced the beginning of the recognition that only the intuition of time need be considered as a priori, back to Descartes' seventeenth-century invention of analytic geometry. And from the author's note to the 1919 reprint: "If this lecture were given to-day, it would certainly mention ... the 'general theory of relativity', contrived after 1909 ..." (see the English translation in [29], p. 120).

${ }^{10}$ Alongside his logical and philosophical work, Heyting continued making technical contributions to intuitionistic mathematics until his retirement. See A. S. Troelstra's appreciation and complete bibliography [110] of Heyting's work.
} 
first noted that coincidence was equivalent to the absurdity of apartness, and then defined the new concept of deviation as the absurdity of coincidence (hence the absurdity of the absurdity of apartness). Intuitionistically, deviation did not imply apartness; but by the theorem just quoted, the absurdity of deviation was equivalent to coincidence.

In $1926 \mathrm{R}$. Wavre published a comparison [133] of classical and "empirist" logical reasoning; the common principles were those he considered to belong to "Brouwerian logic." ${ }^{11}$ A lively discussion followed in print, with contributions by Lévy, Barzin and Errera, Glivenko, Heyting, Borel and Church, among others. ${ }^{12}$ Glivenko [46] incompletely formalized intuitionistic propositional logic; his second version [47] included two axioms proposed to him by Heyting, who would later credit Glivenko with simplifying his own axiomatization. ${ }^{13}$

Heyting's brief 1930 article "On intuitionistic logic" [55] began by endorsing Glivenko's formalization of intuitionistic propositional logic, and went on "to examine how the logic conceived of by Mr. Glivenko is complicated by the addition of the idea of provability Mr. Levy has brought into discussion and that he unduly identifies with the Brouwerian assertion." Instead, wrote Heyting, the Brouwerian assertion of $\mathrm{p}$ (written $\vdash \mathrm{p}$ ) is "It is known how to prove p. ... The idea of an existence of mathematical entities outside our minds must not enter into the proofs." Then, introducing a new formal symbol + for "is provable," he examined in detail the relation between Brouwerian negation and + and observed: "In order to be able to assert $\vdash \neg+$ p, it suffices to reduce to a contradiction the supposition that one can find a construction proving p." Thus it could happen that for some $\mathrm{p}$ "one would have at the same time $\vdash \neg+\mathrm{p}$ and $\vdash \neg \neg$ p. One should abandon every hope of ever settling the question; the problem would be unresolvable." ${ }^{14}$

In 1928 Hilbert and Ackermann's "Principles of Mathematical Logic"(Grundzüge der theoretischen Logik [62]) appeared, with careful axiomatizations of classical propositional and predicate logic; proofs of the completeness and consistency of the first; and a consistency proof and a clear statement of the completeness problem for the second. ${ }^{15}$ One year later Gödel earned his doctorate from the University of Vienna with a proof of the completeness of classical predicate logic. ${ }^{16}$

\footnotetext{
${ }^{11}$ Wavre accepted $(\mathrm{A} \supset \mathrm{B}) \supset(\neg \mathrm{B} \supset \neg \mathrm{A})$ as empirically correct, observing that the converse holds empirically only if B is a negation. He did not assert Heyting's axiom $\neg \mathrm{A} \supset(\mathrm{A} \supset \mathrm{B})$.

${ }^{12}$ Wavre, Borel and Lévy exchanged views in the Revue de Métaphysique et de Morale. Barzin and Errera, Glivenko, and Heyting published in the Bulletin of the Belgian Royal Academy. Church [32], in English, criticised Barzin and Errera's [5]. Details and some translations are in [86]. The history can be found in [54] and [88].

${ }^{13}$ Like Wavre, Glivenko at first omitted the intuitionistic principle that a contradiction implies every statement; also missing from his first attempt was $\mathrm{A} \supset(\mathrm{B} \supset \mathrm{A})$.

${ }^{14}$ Quotations are from the translation of Heyting [55] by Amy L. Rocha, p. 306ff of [86], with slightly different symbolism. The example Heyting used of a possibly unresolvable mathematical question was the Goldbach conjecture, that every even integer (greater than 2) can be expressed as the sum of two prime numbers. While this conjecture remains unresolved, it was not well chosen because its logical form is such that any proof of its double negation would easily yield, by intuitionistic arithmetic, a proof of the conjecture itself. Since Heyting asserted that in general " $\vdash+$ p has exactly the same meaning as $\vdash$ p," not both $\vdash \mathrm{p}$ and $\vdash \neg+\mathrm{p}$ can hold.

${ }^{15}$ The first three editions of Hilbert and Ackermann's very influential textbook were devoted entirely to classical logic. In the fourth German edition, published in 1959, Ackermann added optional sections on intuitionistic logic and strong negation.

${ }^{16}$ For a full discussion of the classical completeness problem, its history and solution, see the article in this volume titled "Gödel's logic" ([120]). There, van Atten and Kennedy present evidence that Gödel was influenced by two lectures Brouwer gave in Vienna in March, 1928. They also quote a passage from Gödel's doctoral dissertation, crediting Brouwer with stressing that the consistency of a formal system did not in itself imply the existence of a model.
} 
Finally, in 1930 Heyting's three fundamental articles "The formal rules of intuitionistic logic" ([56]) and "The formal rules of intuitionistic mathematics" II and III ([57], [58]) appeared in German in the Sitzungsberichte of the Prussian Academy of Sciences. The first of these formalized intuitionistic propositional logic, the second presented formal systems for intuitionistic first-order predicate logic and arithmetic, and the third contained a partial axiomatization of intuitionistic analysis and Brouwer's theory of species (his substitute for classical set theory). Heyting's work, which will be discussed in detail here, was partly anticipated by Glivenko and also by A. Kolmogorov, whose axiomatization [75] of the intuitionistic propositional logic of implication and negation ("minimal logic") and part of intuitionistic first-order predicate logic was published in 1925 in Russian; it does not appear to have been noticed in the West. ${ }^{17}$

In the 1930s Kurt Gödel and Gerhard Gentzen independently observed that classical logic and arithmetic could be effectively translated into their intuitionistic subtheories. Gödel stated, and Gentzen proved, the disjunction and existence properties for intuitionistic formal logic and arithmetic. A decade later Brouwer's work caught the attention of the American logician Stephen Kleene who eventually, with his student Richard Vesley, developed in [74] an efficient axiomatization and a consistency proof for intuitionistic analysis, clarifying its relation to the classical theory. In 1958 Gödel proved the consistency of classical analysis using primitive recursive functions of higher type (the "Dialectica interpretation" [52]; cf. [2], [120]). Four years later Clifford Spector, another Kleene student, used bar recursive functionals for the same purpose in [109], extending to higher types one of the key principles of intuitionistic analysis.

In 1973 Heyting's student Anne S. Troelstra edited (and mostly wrote) the influential survey [112], which was followed in the 1980s by Michael Beeson's [6] and Troelstra and Dirk van Dalen's [118]. Many other logicians have contributed to the metamathematical study of intuitionistic mathematics and logic, among them P. Aczel, E. W. Beth, M. Bezem, J. Diller, M. Dummett, A. G. Dragalin, M. Fitting, M. Fourman, H. Friedman, S. Ghilardi, R. Grayson, S. Hayashi, G. F. van der Hoeven, W. A. Howard, R. Iemhoff, M. de Iongh, D. H. J. de Jongh, G. Kreisel, S. Kripke, M. Krol, H. Läuchli, D. Leivant, V. Lifschitz, M. Löb, I. Loeb, H. Luckhardt, P. Martin-Löf, D. McCarty, G. Mints, I. Moerdijk, J. Myhill, D. Nelson, J. van Oosten, H. Rasiowa, W. Ruitenberg, B. Scarpellini, A. Scedrov, K. Schütte, D. Scott, P. Scowcroft, C. A. Smorynski, A. S. Troelstra, W. Veldman, A. Visser, F. Waaldijk, S. Weinstein and the author. ${ }^{18}$

Brouwer's chair at the University of Amsterdam was held after his death by Heyting, Troelstra and de Jongh in succession. The tradition of doing intuitionistic mathematics in the spirit of Brouwer has been continued in Nijmegen in the Netherlands by Wim Veldman, a student of J. J. de Iongh, who was a student of Brouwer. Two alternative schools of mathematics based on intuitionistic logic are the Russian recursive mathematics of A. A. Markov and his followers, and Errett Bishop's cautious constructive mathematics which is still under development by Douglas Bridges, Fred Richman, Hajime Ishihara and many others. Intuitionistic logic has found modern applications in computer science and category theory.

\footnotetext{
${ }^{17}$ Mancosu, Zach and Badesa [?] includes an analysis of the content of [75].

${ }^{18}$ Here the term "metamathematical" is used loosely to include the study, by constructive or classical means, of formal systems whose intended interpretation is intuitionistic. Brouwer did not long object in principle to formalizing parts of intuitionistic reasoning, but it is unlikely that he would have approved of using classical methods to study the resulting formal systems.
} 


\section{Intuitionistic Propositional Logic}

\subsection{Brouwer's View of Negation as Absurdity}

Brouwer objected equally to the laws of excluded middle and double negation because he interpreted negation not as falsity, but as absurdity. Thus " $p$ or not $p$ " meant "either $p$ is correct or $p$ is contradictory ;" while "if not not $p$, then $p$ " meant that the absurdity of the absurdity of $p$ (in other words, the consistency of $p$ ) implies that $p$ is correct. ${ }^{19}$ In [21] Brouwer thanked Bernays for pointing out that the general law of excluded middle is derivable from the general law of double negation; the converse observation had already appeared in [11], where Brouwer equated the general law of excluded middle with the (untenable) a priori position that every mathematical question is solvable.

Kolmogorov [76] observed that Brouwer's view transforms a negative assertion into a positive one: "Thus the negation of a is transformed into an existential proposition: "There exists a chain of logical inferences that, under the assumption of the correctness of $a$, leads to a contradiction."' Under material implication, which Heyting explicitly accepted, every statement is derivable from any contradiction. Thus, given a particular contradictory assertion $\perp$, the negation of $a$ can be defined as "if $a$ then $\perp$," reducing negation to implication. Nevertheless, all the early intuitionistic formal systems include negation as a primitive symbol.

\subsection{Heyting's Propositional Calculus}

From a historical perspective the most significant formalization of intuitionistic propositional logic was the one published by Heyting in 1930 ([56]). We present it here using Heyting's notation, but omitting the " $\vdash \vdash$ " preceding each axiom. After observing that the intuitionistic logical connectives $\supset, \wedge, \vee, \neg$ are independent of one another (unlike the classical connectives), he proposed as rules of inference:

1.2. If $\mathrm{a}$ and $\mathrm{b}$ are correct formulas, then $\mathrm{a} \wedge \mathrm{b}$ is also correct.

1.3. If $\mathrm{a}$ and $\mathrm{a} \supset \mathrm{b}$ are correct formulas, then $\mathrm{b}$ is also correct.

and as axiom schemata:

2.1. $\cdot \mathrm{a} \supset \mathrm{a} \wedge \mathrm{a}$.

2.11. $\cdot \mathrm{a} \wedge \mathrm{b} \supset \mathrm{b} \wedge \mathrm{a}$.

2.12. $\cdot \mathrm{a} \supset \mathrm{b} \cdot \supset \cdot \mathrm{a} \wedge \mathrm{c} \supset \mathrm{b} \wedge \mathrm{c}$.

2.13. $\cdot \mathrm{a} \supset \mathrm{b} \cdot \wedge \cdot \mathrm{b} \supset \mathrm{c} \cdot \supset \cdot \mathrm{a} \supset \mathrm{c}$.

2.14. $\cdot \mathrm{b} \supset \cdot \mathrm{a} \supset \mathrm{b}$.

2.15. $\cdot \mathrm{a} \wedge \cdot \mathrm{a} \supset \mathrm{b} \cdot \supset \mathrm{b}$.

3.1. $\cdot \mathrm{a} \supset \mathrm{a} \vee \mathrm{b}$.

\footnotetext{
${ }^{19}$ W. P. van Stigt singles out [16], in which Brouwer proves that "absurdity of absurdity of absurdity is equivalent with absurdity," as evidence of his (temporary) willingness to study metamathematical questions. At that time Brouwer was also interested, with Mannoury, in the Significist philosophy which held that it was possible to improve communication by refining language.
} 
3.11. $\cdot a \vee b \supset b \vee a$.

3.12. $\cdot \mathrm{a} \supset \mathrm{c} \cdot \wedge \cdot \mathrm{b} \supset \mathrm{c} \cdot \supset \cdot \mathrm{a} \vee \mathrm{b} \supset \mathrm{c}$.

4.1. $\cdot \neg \mathrm{a} \supset \cdot \mathrm{a} \supset \mathrm{b}$.

4.11. $\cdot \mathrm{a} \supset \mathrm{b} \cdot \wedge \cdot \mathrm{a} \supset \neg \mathrm{b} \cdot \supset \neg \mathrm{a}$.

In each rule or axiom the $\mathrm{a}, \mathrm{b}, \mathrm{c}$ are metavariables in the sense that each one may be replaced, throughout the statement of the rule or axiom, by any well-defined formula.

Heyting defined logical equivalence by

2.01. $\cdot \mathrm{a} \supset \subset \mathrm{b} \cdot=_{\mathrm{D}} \cdot \mathrm{a} \supset \mathrm{b} \cdot \wedge \cdot \mathrm{b} \supset \mathrm{a}$.

and proceeded to sketch proofs for seventy formal theorems. As an example, consider ${ }^{20}$

2.2. $\vdash \cdot \mathrm{a} \wedge \mathrm{b} \supset \mathrm{a}$.

Proof. [2.14] $\vdash \cdot \mathrm{a} \supset \cdot \mathrm{b} \supset \mathrm{a}: \supset$ :

$[2.12] \vdash \cdot \mathrm{a} \wedge \mathrm{b} \supset \cdot \mathrm{b} \supset \mathrm{a} \cdot \wedge \mathrm{b} \cdot \supset[2.15] \mathrm{a}$.

Observe that the last step involves an implicit use of axiom 2.11; the applications of rule 1.3 are also implicit; but the argument is evidently sound, provided the postulates are correct. We single out a few additional numbered results, omitting the proof-sketches (which depend on intermediate theorems). ${ }^{21}$

2.22. $\vdash \cdot \mathrm{a} \wedge \mathrm{b} \supset \mathrm{b}$.

2.26. $\vdash \cdot \mathrm{b} \supset \cdot \mathrm{a} \supset \mathrm{a} \wedge \mathrm{b}$.

4.2. $\vdash \cdot \mathrm{a} \supset \mathrm{b} \cdot \supset \cdot \neg \mathrm{b} \supset \neg \mathrm{a}$.

4.3. $\vdash \cdot \mathrm{a} \supset \neg \neg \mathrm{a}$.

4.31. $\vdash \cdot \neg \mathrm{a} \supset \neg \neg \neg \mathrm{a}$.

4.32. $\vdash \cdot \neg \neg \neg \mathrm{a} \supset \neg \mathrm{a}$.

4.45. $\vdash \cdot \mathrm{a} \vee \neg \mathrm{a} \cdot \supset \cdot \neg \neg \mathrm{a} \supset \mathrm{a}$.

4.46. $\vdash \cdot \neg \mathrm{a} \vee \mathrm{b} \supset \cdot \mathrm{a} \supset \mathrm{b}$.

4.47. $\vdash \cdot \mathrm{a} \vee \mathrm{b} \supset \cdot \neg \mathrm{a} \supset \mathrm{b}$.

4.8. $\vdash \cdot \neg \neg(\mathrm{a} \vee \neg \mathrm{a})$.

4.83. $\vdash \cdot \mathrm{a} \vee \neg \mathrm{a} \supset \neg \mathrm{b} \cdot \supset \neg \mathrm{b}$.

4.9. $\vdash \cdot \mathrm{a} \supset \mathrm{b} \cdot \supset \neg(\mathrm{a} \wedge \neg \mathrm{b})$.

4.91. $\vdash \cdot \mathrm{a} \vee \mathrm{b} \supset \neg(\neg \mathrm{a} \wedge \neg \mathrm{b})$.

4.92. $\vdash \cdot \mathrm{a} \wedge \mathrm{b} \supset \neg(\neg \mathrm{a} \wedge \neg \mathrm{b})$.

\footnotetext{
${ }^{20}$ In Heyting's version the last "a" of the proof was incorrectly printed as "b."

${ }^{21}$ Heyting credited Glivenko with the formal proofs of 4.3 and 4.8, and with the statement of 4.83 .
} 
To show each of his axioms independent of the other ten, Heyting provided eleven interpretations in the style of Bernays, using finite or countably infinite truth tables. To show axiom 2.15 is independent, for example, he used four truth values $0,1,2,3$ (of which only 0 represents "correct") with the tables

\begin{tabular}{|c|c|c|c|c|c|c|c|c|c|c|c|c|c|c|c|c|c|c|c|}
\hline$\supset$ & 0 & 1 & 2 & 3 & $\wedge$ & 0 & 1 & 2 & 3 & V & 0 & 1 & 2 & 3 & $\neg$ & 0 & 1 & 2 & 3 \\
\hline 0 & 0 & 0 & 0 & 0 & 0 & 0 & 1 & 2 & 2 & 0 & 0 & 0 & 0 & 0 & & & $\overline{0}$ & 1 & 1 \\
\hline 1 & 1 & 0 & 1 & 1 & 1 & 1 & 1 & 1 & 1 & 1 & 0 & 1 & 2 & 3 & & & & & \\
\hline 2 & 2 & 0 & 0 & 0 & 2 & 2 & 1 & 2 & 2 & 2 & 0 & 2 & 2 & 2 & & & & & \\
\hline 3 & 2 & 0 & 2 & 0 & 3 & 2 & 1 & 2 & 2 & 3 & 0 & 3 & 2 & 3 & & & & & \\
\hline
\end{tabular}

When a, b, c independently take arbitrary values in $\{0,1,2,3\}$ every axiom but 2.15 takes the value 0 . But when $\mathrm{a}$ is 0 and $\mathrm{b}$ is 3 then $\mathrm{a} \wedge \cdot \mathrm{a} \supset \mathrm{b}$ takes the value $0 \wedge 2=2$, so $\mathrm{a} \wedge \cdot \mathrm{a} \supset \mathrm{b} \cdot \supset \mathrm{b}$ is $2 \supset 3=2 \neq 0$.

The requirement that the two rules of inference lead from correct formulas to correct formulas imposed restrictions on the interpretations. Heyting observed that for rule 1.2 the condition $0 \wedge 0=0$ was necessary and sufficient; while for rule 1.3 it was enough that $0 \supset \mathrm{a}=0$ only when $\mathrm{a}=0$ (so the first column of the table for $\supset$ must have only one 0 representing the value of $0 \supset 0$ ). However, he failed to notice that rule 1.2 was derivable from the other postulates. To see this, suppose $\mathrm{a}$ and $\mathrm{b}$ are correct. Then so are $\mathrm{b} \supset \mathrm{a}$ (by 2.14 with rule 1.3) and $\mathrm{b} \wedge \mathrm{b}$ (by 2.1 with rule 1.3 ). But $\mathrm{b} \supset \mathrm{a} \cdot \supset \cdot \mathrm{b} \wedge \mathrm{b} \supset \mathrm{a} \wedge \mathrm{b}$ is an instance of 2.12 , so $\mathrm{a} \wedge \mathrm{b}$ follows by two applications of rule 1.3.

Finally Heyting provided a three-valued interpretation for which all the axioms and rules are correct, but not $\neg \neg \mathrm{a} \supset \mathrm{a}$ (and hence not $\mathrm{a} \vee \neg \mathrm{a}$, by theorem 4.45 stated above). Note that for Heyting "correctness" was absolute: "The formula a $\supset$ b means in general: "If $\mathrm{a}$ is correct, then $\mathrm{b}$ is also correct'," ${ }^{22}$ from which it follows that if $\mathrm{b}$ is correct, so is $\mathrm{a} \supset \mathrm{b}$ (the sense of axiom 2.14).

As his main sources Heyting listed Peano's Formulaire des Mathématiques, Russell and Whitehead's Principia Mathematica, Hilbert and Ackerman's Grundzüge der theoretischen Logik, and two articles: Bernays' "Axiomatische Untersuchung des Aussagenkalküls der 'Principia Mathematica'" (Math. Zeitschrift 25 (1926) p. 305) and Glivenko's "Sur la logique de M. Brouwer" (Bulletins de l'Academie royale de Belgique $5^{e}$ serie vol. XIV (1928) p. 225). Glivenko's article deserves a closer look, as it both anticipated and was influenced by Heyting's fomalization.

\subsection{Glivenko's Contribution}

Inspired by Brouwer's [16], Valerii Glivenko published two notes in French in the Académie Royale de Belgique in 1928 ([46]) and 1929 ([47]). The first contained an incomplete axiomatization of intuitionistic propositional logic with modus ponens as its only rule of inference, and a formal proof of $\neg \neg(\mathrm{a} \vee \neg \mathrm{a})$ ("a remarkable theorem of Mr. Brouwer" $[47])$.

The second provided four additional axioms, including Heyting's axioms 2.14 and 3.1 (which Glivenko justified intuitively as consequences of the intuitionistically acceptable principle $\mathrm{a} \vee \neg \mathrm{b} \cdot \supset \cdot \mathrm{b} \supset \mathrm{a}) .{ }^{23}$ On the basis of his new axiomatization, which he claimed

\footnotetext{
${ }^{22}$ p. 44 of the German original.

${ }^{23}$ In a footnote Glivenko gave Heyting credit for convincing him of the necessity of these two axioms, and referred to Heyting's "memoir, which will soon appear in the Mathem. Annalen." In fact Brouwer, who had fallen out of favor with Hilbert, was no longer an editor of that journal and Heyting's formalization
} 
to be complete for intuitionistic propositional reasoning, Glivenko proved two metatheorems (known today as "Glivenko's Theorems") relating intuitionistic and classical logic. In his own words: ${ }^{24}$

1. "If a certain expression in the logic of propositions is provable in classical logic, it is the falsity of the falsity of this expression that is provable in Brouwerian logic."

2. "If the falsity of a certain expression in the logic of propositions is provable in classical logic, that same falsity is provable in Brouwerian logic."

To prove the first theorem he used modus ponens with a $\supset \neg \neg$ a (already proved in [46]) to derive the double negation of each of his axioms from the axiom itself, then verified that $\neg \neg \mathrm{b}$ is derivable in his augmented intuitionistic system from $\neg \neg \mathrm{a}$ and $\neg \neg(\mathrm{a} \supset \mathrm{b})$. The second was an easy corollary of the first, using $\neg \neg \neg \mathrm{a} \supset \neg$ a (also proved in [46]).

Glivenko did not address the question whether all his axioms were necessary, but each is provable in Heyting's formalism. Conversely, each of Heyting's axioms is provable from Glivenko's, and Heyting's rule 1.2 follows from Glivenko's axioms by modus ponens (which is Heyting's rule 1.3). From this point of view the two formal systems are equivalent, although each has advantages and disadvantages. In [69], as we shall see, Kleene made a coherent selection of axioms from their union.

\subsection{Kolmogorov's Problem Interpretation}

In 1932 Kolmogorov [76] provided a kind of semantics for intuitionistic logic, as an alternative to Heyting's emphasis on proofs. Taking the notions of (mathematical) "problem" and "solution of a problem" as already understood, he proposed to attach to every expression of the form $\vdash p(a, b, c, \ldots)$ the problem "to give a general method for the solution of $p(a, b, c, \ldots)$ for any single choice of problems $a, b, c, \ldots$. " Here $p(a, b, c, \ldots)$ is a propositional formula with only the atoms $a, b, c, \ldots$ and the propositional connectives have the following interpretation:

If $a$ and $b$ are two problems, then $a \wedge b$ designates the problem "to solve both $a$ and $b$," while $a \vee b$ designates the problem "to solve at least one of the problems $a$ and $b . "$ Furthermore, $a \supset b$ is the problem "to solve $b$ provided that the solution for $a$ is given" or, equivalently, "to reduce the solution of $b$ to the solution of $a . " \quad \ldots$ Correspondingly, $\neg a$ designates the problem "to obtain a contradiction provided that the solution of $a$ is given."

Kolmogorov thus transformed the logical rules and axioms of Heyting's propositional logic into the postulates of an "elementary calculus of problems." 25

These ideas, which Kolmogorov extended to predicate logic (see the next section), anticipated Kleene and Nelson's 1945 realizability interpretation ([68], [97]) of intuitionistic arithmetic. Together with Heyting's proof interpretation they form the basis of what is often called the "Brouwer-Heyting-Kolmogorov (B-H-K) explication" of intuitionistic logic.

appeared instead in the Proceedings of the Prussian Academy of Sciences as [56], [57] and [58]; cf. [122].

${ }^{24}$ The translation is by Amy L. Rocha, p. 301 of [86].

${ }^{25}$ The quotations are from Paolo Mancosu's translation, [86] pp. 328-334, of [76]. 


\section{$3 \quad$ Intuitionistic First-Order Predicate Logic}

\subsection{The Intuitionistic Universal and Existential Quantifiers}

Brouwer accepted universal quantification over the natural numbers, interpreting the statement that every $n$ has a certain property as an incomplete communication of a construction which, applied in a uniform manner to each natural number $n$ as it is generated, verifies the property for that $n$. The statement that there exists an $m$ with a certain property means that a particular number $m$ with the property has been constructed, or that a method for constructing such a number is known. Kolmogorov similarly interpreted a universal statement as a problem requiring a general method for its solution, and an existential statement as the problem of finding a witness. Thus under the B-H-K interpretation the phrase "for every $n$ there is an $m$ " affirms the construction of an effective operation which assigns to each $n$ an appropriate $m$; this is why the axiom of countable choice is generally considered to be intuitionistically correct.

Quantification over more complicated domains is meaningful intuitionistically only if the elements of the domain are legitimate mathematical entities and the structure of the domain admits uniform effective operations. This interpretation clearly distinguishes intuitionistic quantification from the classical case where each domain is assumed to consist of preexisting objects which can be examined simultaneously for the presence or absence of a given property, and the existential quantifier does not entail the production of a witness.

\subsection{Heyting's Predicate Calculus With Identity}

Continuing the work he began in [56] ( $\$ 2.2$ above), in the introduction to [57] Heyting wrote: ${ }^{26}$

To enable the further decomposition into subject and predicate, we follow the path of functional calculus which was first laid down by Russell and Whitehead, and was recently lucidly presented and significantly improved by Hilbert and Ackermann in their book. Above all, the concept of mathematical object must be explained. The real construction of mathematics begins immediately with the treatment of objects (natural numbers, sets, etc.) For the formal treatment, however, it turns out to be convenient to assume in advance general axioms about the relations between objects and about formulas which contain variables for objects....

The fundamental relation between individuals is $\mathrm{p} \equiv \mathrm{q}$, " $\mathrm{p}$ is the same object as q." ... The symbol = will be used for numerical equality and similar concepts (e.g. cardinal equality for sets); this relation can hold between objects which are not identical; ... We also need a special symbol for mathematical identity, which must be defined for each sort of mathematical objects ... For mathematical identity we choose the symbol $\equiv .^{27}$

He attributed to the formula $\mathrm{p} \equiv \mathrm{p}$ a strong sense according to which "we can translate 6.1. $\vdash \vdash 1 \equiv 1$ by ' 1 is an object." " 28 He used the notation $\left(\begin{array}{c}\mathrm{p} \\ \mathrm{x}\end{array}\right)$ a for " "the expression

\footnotetext{
${ }^{26}$ Unless otherwise noted, all translations from the German of [57] and [58] are my own.

${ }^{27}$ In each mathematical application, $=$ and $\equiv$ must be defined for each sort of object in terms of $\equiv$. In arithmetic there is no difference.

${ }^{28}$ This use of $\equiv$ anticipated by decades a similar use of $=$ in the logic of partial terms, cf. [107], [6].
} 
which results from a by replacing the variable $\mathrm{x}$ throughout, everywhere it appears, by the term (Zeichenzusammenstellung) p.'" Building on Hilbert and Ackermann's [62] and his own [56], Heyting developed the formal language of his predicate calculus using a, b, c, .. for sentential variables (Aussagenveränderliche) and p, q, r, .. for object variables (Gegenstandveränderliche), with the relation symbols $\equiv, \varepsilon$, the function application symbol ', and "objects (Gegenstände) I, N, seq" and specifying that the "quantifiers $\left(\begin{array}{c}\mathrm{p} \\ \mathrm{x}\end{array}\right),(\mathrm{x}),(\mathrm{Ex})$, where $\mathrm{x}$ is an object variable, make $\mathrm{x}$ into a bound variable in the part of the formula they influence. Such [an $\mathrm{x}]$ can only be replaced by another object variable."

His "rules of operation" included an inductive definition of "expression" (i.e. wellformed formula) extending the formation rules for $\supset, \wedge, \vee$ and $\neg$ stated more casually in [56], but for one clause of this definition the concept "correct formula" - which itself depended inductively on the axiom schemata and rules of inference, including those of the propositional calculus - was required by his use of $\equiv$ to confer legitimacy on terms; see rule 5.32 below. Heyting did not define "term" but the usage made clear that object variables and constant objects such as I were terms. We list his key new rules, omitting a few obvious conditions on substitution in expressions but including the formation rules and the rules of inference $5.4-5.7:^{29}$

5.3. Every correct (richtige) formula is an expression (Ausdruck).

5.32. From an expression another expression results, if one replaces a propositional variable throughout by the same expression, or an object variable throughout by the same term $\mathrm{p}$, for which $\mathrm{p} \equiv \mathrm{p}$ is a correct formula.

5.34. If $\mathrm{A}$ is an expression, so is $\neg \mathrm{A}$, and conversely.

5.35. If $\mathrm{A}$ is an expression in which $\mathrm{x}$ does not appear as a bound variable, then $\left(\begin{array}{l}\mathrm{p} \\ \mathrm{x}\end{array}\right) \mathrm{A}$, (x) A, (Ex) A are expressions.

5.36. If $\mathrm{A}$ and $\mathrm{B}$ are expressions, so are $\mathrm{A} \supset \mathrm{B}, \mathrm{A} \wedge \mathrm{B}$ and $\mathrm{A} \vee \mathrm{B}$.

5.4. From a correct formula another correct formula results, if one replaces a propositional variable throughout by the same expression, or an object variable throughout by a term $\mathrm{p}$, for which $\mathrm{p} \equiv \mathrm{p}$ is a correct formula.

5.5. If $\mathrm{a} \supset \mathrm{b}$ and $(\mathrm{p}) \cdot\left(\begin{array}{l}\mathrm{p} \\ \mathrm{x}\end{array}\right) \mathrm{a} \supset \subset$ a are correct formulas, then $\mathrm{a} \supset(\mathrm{x}) \mathrm{b}$ is a correct formula.

5.6. If $\mathrm{a} \supset \mathrm{b}$ and $(\mathrm{p}) \cdot\left(\begin{array}{c}\mathrm{p} \\ \mathrm{x}\end{array}\right) \mathrm{b} \supset \subset \mathrm{b}$ are correct formulas, then $(\mathrm{Ex}) \mathrm{a} \supset \mathrm{b}$ is a correct formula.

\footnotetext{
${ }^{29}$ Richard Zach has observed an anomaly in Heyting's definition of "expression." Rule 5.7 (with 5.3) allows $(\mathrm{y}) \cdot\left(\begin{array}{l}\mathrm{y} \\ \mathrm{x}\end{array}\right) \mathrm{A} \supset \subset \mathrm{A}$ to be an expression if $\mathrm{x}$ occurs (only) bound in A, but then no rule guarantees that e.g. $\left(\begin{array}{l}\mathrm{y} \\ \mathrm{x}\end{array}\right) \mathrm{A}$ is an expression. The gap could be filled by extending the "and conversely" in rule 5.34 to rule 5.36 and adding a rule to the effect that if $\left(\begin{array}{c}\mathrm{p} \\ \mathrm{x}\end{array}\right) \mathrm{A},(\mathrm{x}) \mathrm{A}$, or $(\mathrm{Ex}) \mathrm{A}$ is an expression, so is $\mathrm{A}$.
} 
5.7. If in the expression $\mathrm{A}$ the variable $\mathrm{x}$ appears only bound, then $(\mathrm{y}) \cdot\left(\begin{array}{l}\mathrm{y} \\ \mathrm{x}\end{array}\right) \mathrm{A} \supset \subset \mathrm{A}$ is a correct formula.

As axiom schemata, in addition to those of intuitionistic propositional logic extended to the predicate language, Heyting proposed the following. First, the axioms for equality:

6.1. $1 \equiv 1$.

6.11. $\mathrm{p} \equiv \mathrm{p} \wedge \mathrm{q} \equiv \mathrm{q} \supset \mathrm{p} \equiv \mathrm{q} \vee \neg(\mathrm{p} \equiv \mathrm{q})$.

6.12. $\mathrm{q} \equiv \mathrm{p} \supset \mathrm{p} \equiv \mathrm{q}$.

The next group contained axioms asserting that the replacement operator commutes with the propositional connectives, and also

6.2. $\left(\begin{array}{l}\mathrm{x} \\ \mathrm{x}\end{array}\right) \mathrm{a} \supset \subset \mathrm{a}$.

6.21. $\left(\begin{array}{c}\left(\begin{array}{c}\mathrm{q} \\ \mathrm{x}\end{array}\right) \mathrm{p} \\ \mathrm{x}\end{array}\right) \mathrm{a} \supset \subset\left(\begin{array}{l}\mathrm{q} \\ \mathrm{x}\end{array}\right)\left(\begin{array}{l}\mathrm{p} \\ \mathrm{x}\end{array}\right) \mathrm{a}$.

6.26. $\cdot \mathrm{p} \equiv \mathrm{q} \supset \cdot\left(\begin{array}{l}\mathrm{p} \\ \mathrm{x}\end{array}\right) \mathrm{a} \supset\left(\begin{array}{l}\mathrm{q} \\ \mathrm{x}\end{array}\right) \mathrm{a}$.

Heyting then introduced a notation $\mathrm{g}(\overline{\mathrm{x}})$ for "g does not contain $\mathrm{x}$," with the definition 6.01. $\cdot \mathrm{g}(\overline{\mathrm{x}})=_{\mathrm{D}} \cdot(\mathrm{p})\left(\begin{array}{c}\mathrm{p} \\ \mathrm{x}\end{array}\right) \mathrm{g} \equiv \mathrm{g} \cdot \vee \cdot(\mathrm{p}) \cdot\left(\begin{array}{l}\mathrm{p} \\ \mathrm{x}\end{array}\right) \mathrm{g} \supset \subset \mathrm{g} \cdot$

Without commenting on the peculiarity of the disjunctions obtained by literally replacing $g$ throughout this definition by a term or expression, he remarked: "... The first alternative in 6.01 holds, when $\mathrm{g}$ represents an object; the second, if $\mathrm{g}$ is an expression. The introduction of this symbol will make possible the distinction between function and sentence variables." This aspect of Heyting's formalism illustrates its incompatibility with modern treatments like that of Kleene ([69], p. 82), which will be considered later.

Six quantifier axioms completed the predicate calculus:

6.3. (x) a $\supset\left(\begin{array}{l}\mathrm{p} \\ \mathrm{x}\end{array}\right) \mathrm{a}$.

6.31. (x) a $\supset(y)\left(\begin{array}{l}\mathrm{y} \\ \mathrm{x}\end{array}\right) \mathrm{a}$.

6.32. $\cdot(\mathrm{y}) \cdot \mathrm{y} \equiv \mathrm{y} \supset\left(\begin{array}{c}\mathrm{y} \\ \mathrm{x}\end{array}\right) \mathrm{a} \cdot \supset(\mathrm{x}) \mathrm{a}$.

6.5. $\left(\begin{array}{c}\mathrm{p} \\ \mathrm{x}\end{array}\right)$ a $\supset(\mathrm{Ex}) \mathrm{a}$.

6.51. $\cdot \mathrm{a}(\overline{\mathrm{x}}) \supset \cdot(\mathrm{Ex}) \mathrm{a} \supset(\mathrm{x}) \mathrm{a}$.

6.52. $\cdot(\mathrm{x}) \cdot \mathrm{a} \supset \mathrm{b} \cdot \supset \cdot(\mathrm{Ex}) \mathrm{a} \supset(\mathrm{Ex}) \mathrm{b}$. 
The thirty five formal theorems Heyting proved on this basis included

6.77. $\vdash(\mathrm{Ex}) \neg \neg \mathrm{a} \supset \neg \neg(\mathrm{Ex}) \mathrm{a}$.

6.78. $\vdash \neg \neg(\mathrm{x}) \mathrm{a} \supset(\mathrm{x}) \neg \neg \mathrm{a}$.

He remarked that neither converse is provable, observing "For 6.77 this follows easily from the meaning of (Ex)." For 6.78 he used a Brouwerian counterexample, the set A of all infinitely proceeding sequences of digits 1,2 , such that once a 2 is chosen, only 2 may be chosen thereafter. To each (positive) natural number he assigned an element of A as follows. The number 1 is associated with the constant sequence $1111 \ldots, 2$ with the constant sequence $2222 \ldots$, 3 with $1222 \ldots$, 4 with $1122 \ldots$, and so on. Letting the object variable $\mathrm{x}$ range over the elements of $\mathrm{A}$ and taking a to mean " $\mathrm{x}$ is assigned to a natural number," Heyting argued that $(\mathrm{x}) \neg \neg$ a held for this interpretation by the definition of A, while $\neg(\mathrm{x})$ a held by Brouwer's fan theorem, to be discussed in $\S 7$ below. "However by $4.521(\mathrm{x}) \neg \neg \mathrm{a} \wedge \neg(\mathrm{x}) \mathrm{a}$ is incompatible with the converse of 6.78." 30

\section{Intuitionistic Arithmetic}

In the remainder of [57] Heyting applied intuitionistic predicate logic to arithmetic. The language he chose was so arcane that in the interest of comprehension it will not be used here except in this brief discussion from the historical viewpoint.

\subsection{Heyting's Arithmetic}

From the primitive symbols $\varepsilon$ and ', with the intended interpretations of membership and function application respectively, he first constructed formal definitions (or abbreviations) corresponding to the concepts " $\mathrm{x}$ is a single-valued function from $\mathrm{p}$ to $\mathrm{q}$ " and "s belongs to the image of $\mathrm{r}$ under $\mathrm{x}$," then defined mathematical identity between two functions from p to q extensionally in terms of $\equiv$ on the range. Using the constant symbol $\mathrm{N}$ to represent the set of positive natural numbers, and seq for the successor operation, he added axioms asserting that $\mathrm{I}$ is a natural number, successor is a one-to-one function from numbers to numbers, and I is not a successor; also the axiom schema of mathematical induction. He defined numerical equality $=$ and mathematical identity $\equiv$ between numbers to be the same as $\equiv$. Then he gave the primitive recursive defining equations for + and (quantifierfree) explicit definitions for,$-<$ and $>$, and developed the quantifier-free part of this theory. Among the formal theorems he proved was

10.524. $\vdash \mathrm{p}, \mathrm{q} \varepsilon \mathrm{N} \supset \mathrm{p}=\mathrm{q} \vee \mathrm{p}>\mathrm{q} \vee \mathrm{p}<\mathrm{q}$.

Heyting did not draw the conclusion that every expression A involving only I, seq, =, ,,$+->,<$ and the propositional connectives is formally decidable when all individual variables range over $\mathrm{N}$, although he could have shown this by a straightforward induction on the logical form of A. He did not add a symbol and definition for multiplication. That same year (1930), in the Proceedings of the First Congress of Mathematicians of the Slavic Countries held at Warsaw in 1929, M. Presburger [98] published consistency and

\footnotetext{
${ }^{30}$ In [21] p. 256, from which Heyting said he took this example, Brouwer drew a stronger conclusion: that the set described is not effectively enumerable (in the sense that no correlation of distinct natural numbers to all of its elements is possible).
} 
completeness proofs and a decision procedure for a formal system of classical first-order arithmetic with addition but without multiplication. ${ }^{31}$

What is now usually meant by "Heyting's arithmetic" is a first-order formalism with (at least) an individual constant 0 , a unary function symbol $S$ or ' , binary function symbols + and $\cdot$, and axioms in which the individual variables range implicitly rather than explicitly over the natural numbers. The importance of multiplication is that it permits the coding of finite sequences of numbers by numbers, and this in turn makes possible the coding of arithmetic within arithmetic; cf. $\S 5.1$ below. Troelstra [112] included the axioms for all primitive recursive number-theoretic functions, making his version of Heyting's arithmetic an extension of Primitive Recursive Arithmetic PRA and a subtheory of "Heyting's arithmetic of finite types" $\mathbf{H A}^{\omega}$.

\subsection{Kleene's Intuitionistic Arithmetic}

Anticipating Kleene's formalization of intuitionistic analysis, to be discussed in $\S 6$ below, we now present his standard treatment of intuitionistic arithmetic. With minor changes in symbolism and numbering this is the formal system developed and studied in Kleene's fundamental 1952 textbook Introduction to Metamathematics [69], which played the same role for logicians in the third quarter of the twentieth century as Hilbert and Ackermann's [62] in the second. Kleene's language has countably many individual variables $a_{1}, a_{2}, a_{3}, \ldots$, an individual constant 0 , a unary function symbol ', two binary function symbols + and , and the binary predicate symbol $=$. There are no other predicate symbols. Terms and prime formulas are defined inductively as follows:

- 0 is a term.

- Each individual variable is a term.

- If $s$ and $t$ are terms then $s^{\prime},(s+t)$ and $(s \cdot t)$ are terms.

- If $s$ and $t$ are terms then $(s=t)$ is a prime formula.

Every occurrence of a variable $x$ in a term $s$ or $t$ is free in $(s=t)$. Parentheses and the symbol - may be omitted according to the usual mathematical conventions. Formulas are built from prime formulas using $\wedge, \vee, \supset, \neg, \forall, \exists$, and parentheses (omitted by the usual conventions when the scope of a logical symbol is clear without them, as Heyting omitted unnecessary delimiting dots). An occurrence of a variable $x$ in a formula $\mathrm{A}$ is bound if it is within the scope of an existential or universal quantifier, otherwise free.

The only rules of inference are the logical rules R1 - R3 inherited from Kleene's treatment of propositional and predicate logic (compare Heyting's rules 1.3 and $5.5-5.7$ ):

R1. From A and A $\supset$ B, conclude B.

R2. From $\mathrm{C} \supset \mathrm{A}(\mathrm{x})$ where $\mathrm{x}$ does not occur free in $\mathrm{C}$, conclude $\mathrm{C} \supset \forall \mathrm{xA}(\mathrm{x})$.

R3. From $\mathrm{A}(\mathrm{x}) \supset \mathrm{C}$ where $\mathrm{x}$ does not occur free in $\mathrm{C}$, conclude $\exists \mathrm{xA}(\mathrm{x}) \supset \mathrm{C}$.

\footnotetext{
${ }^{31}$ Cf. Kleene's [69], p. 204. Presburger's article is in German but the title of the volume is in Polish. Kleene noted that Hilbert and Bernays ([63], pp. 359ff) adapted Presburger's proof to a less cumbersome classical formalism, and Joan Ross verified the corresponding result for the intuitionistic subsystem of Presburger arithmetic .
} 
The axioms are of three kinds: the logical axiom schemas X1 - X12, the mathematical induction schema X13, and the particular arithmetical axioms X14-X21 (from which the reflexivity, symmetry and transitivity of $=$ follow). For X11 and X12, $\mathrm{A}(\mathrm{x})$ may be any formula and $\mathrm{t}$ any term free for $\mathrm{x}$ in $\mathrm{A}(\mathrm{x})$. For $\mathrm{X} 13, \mathrm{~A}(\mathrm{x})$ may be any formula and $\mathrm{x}$ any variable. For X14 - X21 the a,b,c are three particular distinct individual variables (for example $\mathrm{a}_{1}, \mathrm{a}_{2}, \mathrm{a}_{3}$ ), so these axioms (unlike X13) are formulas rather than schemas. Their universal closures are provable, and the conclusion of X13 can be strengthened to $\forall \mathrm{xA}(\mathrm{x})$ by $\mathrm{R} 2$ since $\mathrm{x}$ is not free in the hypothesis.

$\mathrm{X} 1 . \mathrm{A} \supset(\mathrm{B} \supset \mathrm{A})$.

$\mathrm{X} 2 .(\mathrm{A} \supset \mathrm{B}) \supset((\mathrm{A} \supset(\mathrm{B} \supset \mathrm{C})) \supset(\mathrm{A} \supset \mathrm{C}))$.

X3. $\mathrm{A} \supset(\mathrm{B} \supset \mathrm{A} \wedge \mathrm{B})$.

X4. $\mathrm{A} \wedge \mathrm{B} \supset \mathrm{A}$.

X5. $\mathrm{A} \wedge \mathrm{B} \supset \mathrm{B}$.

X6. A $\supset \mathrm{A} \vee \mathrm{B}$.

X7. $\mathrm{B} \supset \mathrm{A} \vee \mathrm{B}$.

X8. $(\mathrm{A} \supset \mathrm{C}) \supset((\mathrm{B} \supset \mathrm{C}) \supset(\mathrm{A} \vee \mathrm{B} \supset \mathrm{C}))$.

X9. $(\mathrm{A} \supset \mathrm{B}) \supset((\mathrm{A} \supset \neg \mathrm{B}) \supset \neg \mathrm{A})$.

$\mathrm{X} 10 . \neg \mathrm{A} \supset(\mathrm{A} \supset \mathrm{B})$.

$\mathrm{X} 11 . \forall \mathrm{xA}(\mathrm{x}) \supset \mathrm{A}(\mathrm{t})$.

X12. $\mathrm{A}(\mathrm{t}) \supset \exists \mathrm{xA}(\mathrm{x})$.

X13. $\mathrm{A}(0) \wedge \forall \mathrm{x}\left(\mathrm{A}(\mathrm{x}) \supset \mathrm{A}\left(\mathrm{x}^{\prime}\right)\right) \supset \mathrm{A}(\mathrm{x})$.

X14. $\left(a^{\prime}=b^{\prime}\right) \supset(a=b)$.

X15. $\neg\left(\mathrm{a}^{\prime}=0\right)$.

X16. $(\mathrm{a}=\mathrm{b}) \supset((\mathrm{a}=\mathrm{c}) \supset(\mathrm{b}=\mathrm{c}))$.

X17. $(\mathrm{a}=\mathrm{b}) \supset\left(\mathrm{a}^{\prime}=\mathrm{b}^{\prime}\right)$.

$\mathrm{X} 18 .(\mathrm{a}+0)=\mathrm{a}$.

X19. $\left(\mathrm{a}+\mathrm{b}^{\prime}\right)=(\mathrm{a}+\mathrm{b})^{\prime}$.

$\mathrm{X} 20 .(\mathrm{a} \cdot 0)=0$.

$\mathrm{X} 21 .\left(\mathrm{a} \cdot \mathrm{b}^{\prime}\right)=(\mathrm{a} \cdot \mathrm{b})+\mathrm{a}$.

After developing an unambiguous coding in this theory for finite sequences of natural numbers, Kleene proved that symbols and defining equations for any number of primitive recursive functions could be conservatively added to the system. He went on to give a careful exposition of the negative results of Gödel, Church and Rosser for first-order intuitionistic number theory as well as for the classical theory which results when Heyting's 
axiom schema X10 is replaced by $\neg \neg \mathrm{A} \supset \mathrm{A}$. We state these results in the next section; for details, and also for historical evidence of Brouwer's influence on Gödel, see van Atten and Kennedy's [120] in this volume.

Kleene's formalization of pure intuitionistic first-order predicate logic without equality is obtained by allowing only individual variables as terms, allowing as prime formulas only proposition letters and expressions formed from $n$-place predicate letters by filling the places with occurrences of variables, and omitting the arithmetical axioms X13 - X21. From now on we refer to this theory, based on rules R1 - R3 and axiom schemas X1 $\mathrm{X} 12$, as "intuitionistic predicate logic" IQC. By "intuitionistic propositional logic" IPC we mean the subtheory of IQC with only proposition letters as prime formulas, only the rule R1 and only the axiom schemas X1 - X10. ${ }^{32}$

\section{$5 \quad$ Proof Theory of Intuitionistic Logic and Arithmetic}

Classical propositional and predicate logic, and a version of classical (Presburger) arithmetic, can be obtained from Heyting's formal systems simply by replacing axiom schema 4.1 by either the law of excluded middle or the law of double negation; then 4.1 becomes a theorem. Kleene's formalisms have the same property. Thus intuitionistic logic and arithmetic are proper subtheories of the corresponding classical theories.

Classical propositional logic can be formalized using only two logical connectives $(\wedge$ and $\neg$, for example), from which the others are definable. Classical predicate logic needs only one quantifier (for example, $\forall$ ) from which the other is definable using negation. ${ }^{33}$ The intuitionistic logical language is thus more expressive than the classical, allowing finer distinctions. But is intuitionistic logic essentially weaker than classical logic? This question was answered in stages, and the answer is a firm "no."

\subsection{Equiconsistency and the Negative Translations}

Wishing to defend the consistency of the classical law of excluded middle, Kolmogorov [75] gave a nearly complete axiomatization of minimal logic, the intuitionistic propositional logic of $\supset$ and $\neg .{ }^{34}$ Focusing on the importance of what is now called "stability under double negation," Kolmogorov showed that if a proposition b in this language could be proved using also the classical schema $\neg \neg \mathrm{a} \supset \mathrm{a}$, then the proposition $\mathrm{b}^{k}$ obtained from b by doubly negating each of its prime components could be proved intuitionistically. This transformation effectively reduced all of classical propositional logic to Kolmogorov's fragment of minimal logic.

Glivenko [46], [47] also reduced the consistency of classical propositional logic to that of the intuitionistic theory. If some formula and its negation were both classically provable, then by Glivenko's theorems both the double negation and the negation of that formula would be provable intuitionistically, so intuitionistic propositional logic would be inconsistent. Glivenko's Theorems, as stated in $\S 2.3$, do not hold for intuitionistic predicate logic, although Gödel [51] showed that they do for the intermediate logic obtained by adding as

\footnotetext{
${ }^{32}$ Troelstra and van Dalen [118] used the acronyms IQC and IPC for different, but equivalent, axiomatizations of intuitionistic predicate and propositional logic respectively.

${ }^{33}$ Classically (but not intuitionistically) $\mathrm{A} \vee \mathrm{B}$ is equivalent to $\neg(\neg \mathrm{A} \wedge \neg \mathrm{B}), \mathrm{A} \supset \mathrm{B}$ is equivalent to $\neg(\mathrm{A} \wedge \neg \mathrm{B})$, and $\exists \mathrm{xA}(\mathrm{x})$ is equivalent to $\neg \forall \mathrm{x} \neg \mathrm{A}(\mathrm{x})$.

${ }^{34}$ Like Glivenko [46], he omitted Heyting's axiom 4.1; cf. [?].
} 
axiom schema the converse of Heyting's theorem $6.78 .{ }^{35}$

Gödel [51] and Gentzen [42] independently completed the picture by faithfully translating classical predicate logic into the fragment of intuitionistic predicate logic using only the logical symbols $\wedge, \neg, \forall$, by recursion on the inductive definition of formula (i.e. by "formula induction"). The Gödel translation $\mathrm{p}^{\mathrm{g}}$ of a prime formula $\mathrm{p}$ may be taken to be $\neg \neg \mathrm{p}$, and compound formulas are translated as follows:

$$
\begin{gathered}
(\mathrm{a} \wedge \mathrm{b})^{\mathrm{g}} \equiv_{\mathrm{D}} \mathrm{a}^{\mathrm{g}} \wedge \mathrm{b}^{\mathrm{g}}, \quad(\mathrm{a} \supset \mathrm{b})^{\mathrm{g}} \equiv_{\mathrm{D}} \neg\left(\mathrm{a}^{\mathrm{g}} \wedge \neg \mathrm{b}^{\mathrm{g}}\right), \quad(\mathrm{a} \vee \mathrm{b})^{\mathrm{g}} \equiv_{\mathrm{D}} \neg\left(\neg \mathrm{a}^{\mathrm{g}} \wedge \neg \mathrm{b}^{\mathrm{g}}\right), \\
(\neg \mathrm{a})^{\mathrm{g}} \equiv_{\mathrm{D}} \neg \mathrm{a}^{\mathrm{g}}, \quad(\forall \mathrm{x} \mathrm{a})^{\mathrm{g}} \equiv_{\mathrm{D}} \forall \mathrm{x} \mathrm{a}^{\mathrm{g}} \quad \text { and } \quad(\exists \mathrm{x} \mathrm{a})^{\mathrm{g}} \equiv_{D} \neg \forall x \neg a^{g} .
\end{gathered}
$$

It is not hard to show that a $\supset \subset \mathrm{a}^{\mathrm{g}}$ is classically provable for every formula $\mathrm{a}$, and that the formula $\mathrm{a}$ is provable classically if and only if $\mathrm{a}^{\mathrm{g}}$ is provable intuitionistically. The Gentzen translation is similar except that implications are translated by implications.

The negative translation applies also to arithmetic, where prime formulas (equalities) can be taken as their own translations. In intuitionistic arithmetic every formula containing no disjunction and no existential quantifier is stable under double negation, in the sense that the formula and its double negation are provably equivalent.

Kleene ([69] p. 497) remarked that van Dantzig [123] suggested "that it may be possible to interpret practically the whole of classical mathematics within this stable part of the intuitionistic system;" much later P. Krauss ([77], unpublished) carried out part of this plan. In essence, one simply treats the combinations $\forall \neg \neg$ and $\neg \neg \exists$ as classical quantifiers, and the Gödel translation of $\vee$ as classical disjunction, within the intuitionistic language; cf. [93]. The double negation following the universal quantifier is needed to break the uniform dependence of the quantified expression on the variable in question.

The negative translation established the proof-theoretic equivalence of a variety of classical theories with their intuitionistic subtheories. In 1932 Gödel [48] showed that if classical first-order arithmetic with addition and multiplication is $\omega$-consistent (that is, if for no formula $\mathrm{A}(\mathrm{x})$ are $\neg \forall \mathrm{xA}(\mathrm{x}), \mathrm{A}(\mathbf{0}), \mathrm{A}(\mathbf{1}), \mathrm{A}(\mathbf{2}), \ldots$ all provable), then it is incomplete in the sense that there is a sentence $\mathrm{E}$ such that neither $\mathrm{E}$ nor $\neg E$ is provable; and if it is simply consistent then the sentence expressing its consistency is unprovable. These are Gödel's famous First and Second Incompleteness Theorems. In 1936 Church [33], [34] proved that classical predicate logic and classical arithmetic (with + and - and assuming $\omega$-consistency) are undecidable in the sense that there is no recursive procedure for deciding whether or not a given sentence is provable. In the same year Rosser [102] showed that simple consistency was a strong enough assumption for both Gödel's and Church's theorems. These results hold also for the corresponding intuitionistic subtheories. ${ }^{36}$

Simple consistency of classical (hence also of intuitionistic) predicate logic was established by Hilbert and Ackermann using two-valued truth tables with a one-element domain. After proving his incompleteness theorems, Gödel remarked that it might still be possible to establish the consistency of arithmetic using methods which were finitary in a wider sense, but not formalizable in arithmetic. By rethinking the nature of logical derivation and admitting a rule of inference (the $\omega$-rule) with countably infinitely

\footnotetext{
${ }^{35}$ Cf. Troelstra [112] p. 93, where this converse is called "double negation shift." Troelstra notes that it also justifies the negative translation of the axiom schema of countable choice. However, as Heyting's counterexample ( $\S 3.2$ above) showed, double negation shift fails intuitionistically when the universal quantifier ranges over choice sequences.

${ }^{36}$ See [120] for a fuller discussion of Gödel's, Church's and Rosser's theorems.
} 
many premises, Gentzen [43] succeeded in constructing a consistency proof for a system of arithmetic for which consistency and $\omega$-consistency were equivalent by fiat. His method applied directly to intuitionistic as well as to classical $\omega$-arithmetic, by a simple structural restriction which also marked the difference between his formal systems for intuitionistic and classical logic. This brings us to the next topic.

\subsection{Constructive Disjunction and Existence}

The power of intuitionistic logic lies in the effectiveness or constructiveness of its conclusions. Within reason, negative translations of nonconstructive axioms can be added to an intuitionistic theory without losing this effectiveness; and although the presence of all those double negations may seem clumsy and unnecessary to a classical mathematician, there is a practical value to this approach.

The constructive nature of intuitionistic logic is exemplified by the fact that if $\mathrm{A} \vee \mathrm{B}$ is provable and closed (i.e., contains no free occurrences of individual variables) then at least one of A,B must be provable. For intuitionistic propositional logic this disjunction property [DP] was stated without proof by Gödel [49] in 1932 and proved by Gentzen [43] in 1935 (see the next subsection); Gentzen also proved it for intuitionistic predicate logic. Implicit in Gentzen's work and in Schütte [104] was the existence property:

$[\mathrm{EP}]$ : If $\exists \mathrm{x} \mathrm{A}(\mathrm{x})$ is a closed theorem, then $\mathrm{A}(\mathrm{t})$ is a theorem for some term $\mathrm{t}$.

for intuitionistic predicate logic. ${ }^{37}$ The following strong existence property for intuitionistic arithmetic is due to Kleene ([68] p. 115 and Corollary 2(b) to Theorem 62 of [69], with the discussion following) and was reproved by Harrop [53], extending a partial result by Rasiowa [99]:

$[\mathrm{EPN}]$ : If $\exists \mathrm{x} \mathrm{A}(\mathrm{x})$ is a closed theorem, then $\mathrm{A}(\mathbf{n})$ is a theorem for some numeral $\mathbf{n}$.

Here the numerals are $0,0^{\prime}, 0^{\prime \prime}, 0^{\prime \prime \prime}, \ldots{ }^{38}$

\subsection{Gentzen's Contribution}

All the formal systems we have considered so far are Hilbert-style, with many axioms and few rules of inference. Proofs and derivations are linear, with each step possibly depending logically on one or more earlier steps. For some uses a natural deduction system is preferable, with few axioms and many rules of inference; derivations are presented as trees, clarifying the dependency relations.

Gentzen [43] introduced a new kind of formal system for propositional and predicate logic, in which antecedents (assumptions) as well as consequents were listed at each step. Each step in a Gentzen-style derivation was a sequent of the form $\mathrm{A}_{1}, \ldots, \mathrm{A}_{m} \rightarrow \mathrm{B}_{1}, \ldots \mathrm{B}_{s}$ where $\mathrm{A}_{1}, \ldots, \mathrm{A}_{m}$ and $\mathrm{B}_{1}, \ldots \mathrm{B}_{s}$ were formulas, and for the intuitionistic system $s$ must

\footnotetext{
${ }^{37}$ Since individual variables are the only terms of pure predicate logic, it follows that if $\exists x A(x)$ is a closed theorem of pure intuitionistic predicate logic, so is $\forall x A(x)$. Kleene [70] observes: "This result was evidently communicated by Gentzen to Heyting in advance of the publication of ([43]) ..."

${ }^{38}$ Kleene proved the stronger result that if $\forall \mathrm{x} \exists \mathrm{yA}(\mathrm{x}, \mathrm{y})$ is a closed theorem of $\mathbf{H A}$, then there is a recursive function $\varphi$ such that for every $n$ : if $\varphi(n)=m$ then $\mathrm{A}(\mathbf{n}, \mathbf{m})$ is provable.

In arithmetic, disjunction can be defined from existential quantification by $\mathrm{A} \vee \mathrm{B} \equiv_{\mathrm{D}} \exists x[(x=0 \supset$ $A) \wedge(\neg(x=0) \supset B)]$ so the existence property entails the disjunction property. In 1975 H. Friedman [40] showed that the converse holds also, for any recursively enumerable extension of $\mathbf{H A}$.
} 
always be $\leq 1$. The only axiom schema was $\mathrm{C} \rightarrow \mathrm{C}$. Rules of inference were of two kinds: rules for introducing logical symbols in the antecedent and consequent of a sequent, and structural or bookkeeping rules. We give the rules for introducing negation: ${ }^{39}$

$$
\begin{array}{ll}
\text { (in consequent): } & \text { From } \mathrm{A}, \Gamma \rightarrow \Theta \text { conclude } \Gamma \rightarrow \Theta, \neg \mathrm{A} . \\
\text { (in antecedent): } & \text { From } \Gamma \rightarrow \Theta, \mathrm{A} \text { conclude } \neg \mathrm{A}, \Gamma \rightarrow \Theta .
\end{array}
$$

Here $\Gamma$ and $\Theta$ are lists of formulas, with $\Theta$ empty for the intuitionistic system. The reason for the restriction on $s$ (which follows from the restrictions on $\Theta$ ) for the intuitionistic system becomes clear if we consider that for $s>1$ the sequent $\mathrm{A}_{1}, \ldots, \mathrm{A}_{m} \rightarrow \mathrm{B}_{1} \vee \ldots \vee \mathrm{B}_{s}$ could be derived from $\mathrm{A}_{1}, \ldots, \mathrm{A}_{m} \rightarrow \mathrm{B}_{1}, \ldots, \mathrm{B}_{s}$ and so $\rightarrow \mathrm{A} \vee \neg \mathrm{A}$ would be provable from $\rightarrow \mathrm{A}, \neg \mathrm{A}$ which follows from $\mathrm{A} \rightarrow \mathrm{A}$ by the unrestricted first negation-introduction rule.

The only introduction rule for which the logical form of the hypothesis or hypotheses could not be determined from the logical form of the conclusion was the cut rule, which for the intuitionistic system read as follows:

$$
\text { From } \Delta \rightarrow \mathrm{A} \text { and } \mathrm{B}, \Gamma \rightarrow \Theta \text { conclude } \mathrm{A} \supset \mathrm{B}, \Delta, \Gamma \rightarrow \Theta \text {. }
$$

Gentzen proved that every (intuitionistic or classical) derivation in his system could be reduced to a normal form, in which the cut rule was not used, and that every formula occurring in any sequent of a normal derivation was a subformula of some formula occurring in the antecedent or consequent of the last sequent. This remarkable result is Gentzen's Hauptsatz.

The disjunction property for intuitionistic logic is an immediate consequence. In any Gentzen-style proof of a sentence $\mathrm{E}$, the last sequent would be $\rightarrow \mathrm{E}$; and if $\mathrm{E}$ was $\mathrm{A} \vee \mathrm{B}$ then the previous sequent in a normal intuitionistic derivation must have been either $\rightarrow \mathrm{A}$ or $\rightarrow \mathrm{B}$. To see that the disjunction property fails for classical propositional logic, consider $\mathrm{P} \vee \neg \mathrm{P}$ where $\mathrm{P}$ is prime. Since propositional logic is closed under substitution, the provability of $\mathrm{P}$ or of $\neg \mathrm{P}$ would yield inconsistency; yet $\mathrm{P} \vee \neg \mathrm{P}$ is classically provable.

\subsection{Admissible Rules and Intermediate Logics}

The disjunction and existence properties are special cases of an important structural difference, which has recently been illuminated, between intuitionistic and classical logic. The difference concerns the distinction between derivable and nonderivable admissible rules of inference.

A logical rule is a schema, a shorthand notation for infinitely many particular inferences. If $\mathcal{S}$ is a Hilbert-style formal system closed under substitution and if $\mathrm{A}_{1}, \ldots, \mathrm{A}_{\mathrm{k}}, \mathrm{B}$, are formulas, the logical rule

From $\mathrm{A}_{1}, \ldots, \mathrm{A}_{\mathrm{k}}$ conclude $\mathrm{B}$.

means that if $\sigma\left(\mathrm{A}_{1}\right), \ldots, \sigma\left(\mathrm{A}_{\mathrm{k}}\right), \sigma(\mathrm{B})$ are obtained by a uniform substitution of particular formulas for the predicate letters occurring in $\mathrm{A}_{1}, \ldots, \mathrm{A}_{\mathrm{k}}, \mathrm{B}$, and if $\sigma\left(\mathrm{A}_{1}\right), \ldots, \sigma\left(\mathrm{A}_{\mathrm{k}}\right)$ have already been deduced in $\mathcal{S}$ from assumptions $\Gamma$, then $\sigma(\mathrm{B})$ can also be deduced in $\mathcal{S}$ from $\Gamma$. In predicate logic there may be some restrictions on the free variables. The

\footnotetext{
${ }^{39}$ We follow Kleene's presentation of Gentzen's system G1 in [69] §77. For more detail see Kleene [69] $\S \S 77-79$ and von Plato's chapter on Gentzen's logic in this volume.
} 
rule is called admissible for $\mathcal{S}$ if, for every substitution $\sigma$, if $\sigma\left(\mathrm{A}_{1}\right), \ldots, \sigma\left(\mathrm{A}_{\mathrm{k}}\right)$ are closed theorems of $\mathcal{S}$, then $\sigma(\mathrm{B})$ is also provable in $\mathcal{S}$; the standard notation for this situation is

$$
\mathrm{A}_{1}, \ldots, \mathrm{A}_{\mathrm{k}} \sim_{\mathcal{S}} \mathrm{B}
$$

The rule is called derivable in $\mathcal{S}$ if the conclusion is deducible in $\mathcal{S}$ from the hypotheses, i.e. if $\mathrm{A}_{1}, \ldots, \mathrm{A}_{\mathrm{k}} \vdash_{\mathcal{S}} \mathrm{B}$.

For pure classical logic there is essentially no difference; every admissible rule of CPC is classically derivable, and every nonderivable admissible rule of $\mathbf{C Q C}$ is vacuous in the sense that there is no substitution $\sigma$ which converts each hypothesis into a closed theorem (cf. [39]). Intuitionistically the situation is more interesting.

One of the basic tenets of Brouwer's philosophy is that no formal system can adequately represent intuitionistic reasoning, even about mathematics. The purpose of intuitionistic logic is to clarify some general kinds of reasoning which can always be supported by correct constructions. From the intuitionistic viewpoint, a rule of the form

$$
\text { From } A_{1}, \ldots, A_{k} \text { conclude } B \text {. }
$$

is admissible for a formal system $\mathcal{S}$ if there exists an algorithm which transforms any given $k$-tuple $\Pi_{1}, \ldots, \Pi_{k}$, such that each $\Pi_{i}$ is a proof in $\mathcal{S}$ of a sentence of the form $\sigma\left(\mathrm{A}_{i}\right)$, into a proof in $\mathcal{S}$ of $\sigma(\mathrm{B})$. The algorithm has no work to do in the case of a substitution $\sigma$ for which some $\sigma\left(\mathrm{A}_{i}\right)$ is unprovable in $\mathcal{S}$, even if $\sigma\left(\mathrm{A}_{i}\right)$ can be established by other intuitionistically correct means.

On the other hand, the rule is derivable in $\mathcal{S}$ if and only if there exists a general deduction-schema in $\mathcal{S}$ establishing that $\mathrm{B}$ follows in $\mathcal{S}$ from $\mathrm{A}_{1}, \ldots, \mathrm{A}_{\mathrm{k}}$. This is evidently a stronger requirement on two counts: it applies to every $(k+1)$-tuple of formulas of the form $\mathrm{A}_{1}, \ldots, \mathrm{A}_{\mathrm{k}}, \mathrm{B}$, and it insists that $\mathcal{S}$ itself must provide an algorithm for establishing the correctness of the inference.

The disjunction property, when stated as

\section{From $\mathrm{A} \vee \mathrm{B}$ conclude $\mathrm{A}$ or $\mathrm{B}$}

for A, B closed formulas, is an important admissible propositional rule of intuitionistic logic which is completed constructively by an algorithm for producing, from an arbitrarily given proof of any sentence of the form $\mathrm{A} \vee \mathrm{B}$, either a proof of $\mathrm{A}$ or a proof of $\mathrm{B}$. In its presence every other admissible propositional rule can be stated with a unique conclusion.

Two early examples of admissible nonderivable propositional rules of intuitionistic logic are the "independence of premise" rule:

$$
\neg \mathrm{A} \supset \mathrm{B} \vee \mathrm{C} \sim(\neg \mathrm{A} \supset \mathrm{B}) \vee(\neg \mathrm{A} \supset \mathrm{C}) .
$$

and Mints' rule ([90]):

$$
(\mathrm{A} \supset \mathrm{B}) \supset \mathrm{A} \vee \mathrm{C} \sim((\mathrm{A} \supset \mathrm{B}) \supset \mathrm{A}) \vee((\mathrm{A} \supset \mathrm{B}) \supset \mathrm{C}) .
$$

The disjunction rule evidently works together with the other rules to strengthen their conclusions. By generalizing Mints' rule, A. Visser and D. H. J. de Jongh identified a recursively enumerable sequence of successively stronger admissible nonderivable rules of intuitionistic propositional logic and conjectured that they form a basis for all such rules in the sense that every admissible rule of propositional logic is derivable from them together 
with the disjunction property and modus ponens. "Visser's rules" are all the rules of the form

$$
(\mathrm{A} \supset \mathrm{B} \vee \mathrm{C}) \vee \mathrm{D} \sim \bigvee_{\mathrm{i}=1}^{\mathrm{n}}\left(\mathrm{A} \supset \mathrm{E}_{\mathrm{i}}\right) \vee(\mathrm{A} \supset \mathrm{B}) \vee(\mathrm{A} \supset \mathrm{C}) \vee \mathrm{D} .
$$

where $n$ is a positive integer and $\mathrm{A}$ is $\bigwedge_{\mathrm{i}=1}^{\mathrm{n}}\left(\mathrm{E}_{\mathrm{i}} \rightarrow \mathrm{F}_{\mathrm{i}}\right)$. Using results of Ghilardi [45], in 2001 R. Iemhoff [65] succeeded in proving their conjecture. Since Visser's comprehensive 1999 survey [131] contained a proof that the admissible propositional rules of intuitionistic arithmetic are the same as those of intuitionistic propositional logic, Iemhoff's result applied also to Heyting arithmetic.

Because its hypothesis as well as its conclusion depends on the provability relation, a rule which is admissible for one formal system may fail for an extension of that system by additional axiom schemas. An intermediate propositional logic is any consistent collection of propositional formulas containing all the theorems of intuitionistic logic and closed under modus ponens and substitution (of arbitrary formulas for propositional variables). Every intermediate propositional logic is contained in classical propositional logic.

Gödel [50] introduced an infinite sequence of intermediate logics in order to prove that intuitionistic propositional logic had no finite truth-table interpretation. The $n$th Gödel logic $G_{n}$ is axiomatized over IPC by the two schemas

$$
\bigvee_{i=1}^{n}\left(\bigwedge_{j=1}^{i-1} A_{j} \supset A_{i}\right) \quad \text { and } \quad(A \supset B) \vee(B \supset A)
$$

The Visser rules are derivable in $G_{n}$ but the disjunction property does not hold. Gabbay and deJongh [41] found an infinite sequence of finitely axiomatizable intermediate logics $D_{n}$ with the disjunction property, such that the first $n+1$ Visser rules (but not the $n+2$ nd) are admissible for $D_{n}$. Finally Iemhoff [66] proved that intuitionistic propositional logic is the only intermediate logic with the disjunction property which is closed under all the Visser rules.

The nonderivable admissible predicate rules of intuitionistic predicate logic have not been completely characterized, but they are known to form a complete $\Pi_{2}^{0}$ set and to include Markov's rule for decidable predicates:

$$
\forall \mathrm{x}(\mathrm{A}(\mathrm{x}) \vee \neg \mathrm{A}(\mathrm{x})) \wedge \neg \forall \mathrm{x} \neg \mathrm{A}(\mathrm{x}) \quad \sim \quad \exists \mathrm{xA}(\mathrm{x}) .
$$

and an additional independence of premise rule:

$$
\forall \mathrm{x}(\mathrm{A}(\mathrm{x}) \vee \neg \mathrm{A}(\mathrm{x})) \wedge(\forall \mathrm{xA}(\mathrm{x}) \rightarrow \exists \mathrm{yB}(\mathrm{y})) \quad \sim \quad \exists \mathrm{y}(\forall \mathrm{xA}(\mathrm{x}) \rightarrow \mathrm{B}(\mathrm{y})) .
$$

where y must not occur free in $\forall \mathrm{xA}(\mathrm{x})$. These rules also hold for sentences of intuitionistic arithmetic. Markov's rule and the decidability (hence also the stability under double negation) of quantifier-free arithmetical formulas give the important result (Kreisel [78]) that classical and intuitionistic arithmetic prove the same formulas of the form $\forall \mathrm{x} \exists \mathrm{yA}(\mathrm{x}, \mathrm{y})$ with $\mathrm{A}(\mathrm{x}, \mathrm{y})$ quantifier-free, so they have the same provably recursive functions. ${ }^{40}$

An admissible rule with no hypotheses is simply a logical theorem. The propositional logic of intuitionistic arithmetic consists of those formulas in the language of propositional

\footnotetext{
${ }^{40} \mathrm{~A} \Pi_{2}^{0}$ relation is one which can be expressed in $\forall \exists$ form, with a recursive scope. A relation is complete $\Pi_{2}^{0}$ if it is $\Pi_{2}^{0}$, and every $\Pi_{2}^{0}$ relation is recursive in it. The standard complete $\Pi_{2}^{0}$ relation $R(e)$ is " $e$ is the Gödel number of a total recursive function," or equivalently "for every $n$ there is an $m$ which is the Gödel number of a computation of the value of the eth partial recursive function on the argument $n$."
} 
logic, all of whose arithmetical substitution instances are provable in Heyting arithmetic. De Jongh [67] proved that the propositional logic of Heyting arithmetic is IPC. To quote Visser [131], de Jongh actually established "a much stronger result, viz., that the logic of relative interpretations in HA is Intuitionistic Predicate Logic ..." 41 His proof was a clever combination of recursive realizability and Kripke semantics, two interpretations of intuitionistic logic and mathematics which will be introduced in the next section.

\section{Interpretations of Intuitionistic Logic and Arithmetic}

After the formalization of intuitionistic logic and Gödel's completeness proof for classical logic, the search for classical interpretations of intuitionistic propositional and predicate logic began. This semantic approach has produced two related interpretations which are sound and complete for IPC and IQC: algebraic semantics and Beth-Kripke semantics. The constructive viewpoint is probably best represented by Kleene and Nelson's realizability semantics for HA, for which it is sound but not complete. We discuss these three interpretations here in historical order.

\subsection{Algebraic and Topological Interpretations}

According to Troelstra [116] the first semantics for IPC, "due to the work of S. Jaskowski, M. H. Stone, A. Tarski, G. Birkhoff, T. Ogasawara in the years 1936-1940," was an algebraic interpretation in which elements of a Heyting algebra were assigned as truth values to propositions. A Heyting algebra is a lattice with a maximum and a minimum element and an operation $\supset$ satisfying the condition $\mathrm{a} \wedge \mathrm{b} \leq \mathrm{c}$ if and only if $\mathrm{a} \leq \mathrm{b} \supset \mathrm{c}$.

The topological interpretation, developed for IQC as well by Mostowski ([95]) and by Rasiowa and Sikorski ([100], [101]), uses the algebra of open sets of a topological space $T$ under the partial ordering $\subseteq$, with $G \cup H$, Int $(G \cap H)$ and $\operatorname{Int}(H \cup(T \backslash G))$ respectively interpreting $\mathrm{a} \vee \mathrm{b}, \mathrm{a} \wedge \mathrm{b}, \mathrm{a} \supset \mathrm{b}$ when $\mathrm{a}$ is interpreted by $G$ and $\mathrm{b}$ by $H$. The logic then requires that $\neg$ a be interpreted by $\operatorname{Int}(T \backslash G)$. For the extension to predicate logic $\exists$ is interpreted by union, and $\forall$ by the interior of the intersection. Soundness guarantees that each closed theorem is always interpreted by $T$. Completeness asserts that to each unprovable sentence E of IPC or IQC there corresponds a topological space in which E does not take $T$ as value. The topological interpretation was later extended to (subsystems of) intuitionistic analysis by Scott ([105], [106]), Krol' ([84]) and others.

\subsection{Recursive Realizability and Church's Thesis}

Kleene's number-realizability interpretation [68] for intuitionistic arithmetic was inspired by a passage in Hilbert and Bernays [63], but it can easily be understood as a recursive implementation of the B-H-K explication of the logical connectives and quantifiers. It used the fact that an ordered pair of natural numbers $(n, m)$ can be (primitive) recursively coded by a natural number $k$ in such a way that $n$ and $m$ (call them $(k)_{0}$ and $(k)_{1}$, respectively) can be recovered (primitive) recursively from $k^{42}$ It also used the recursively enumerable

\footnotetext{
${ }^{41} \mathrm{~A}$ relative interpretation is an interpretation of a theory over a domain which can be represented by a formula $\mathrm{D}(\mathrm{x})$ for which the theory proves $\exists \mathrm{xD}(\mathrm{x})$. Then $\forall \mathrm{x}\left(\mathrm{D}(\mathrm{x}) \supset \mathrm{A}^{\prime}(\mathrm{x})\right)$ and $\exists \mathrm{x}\left(\mathrm{D}(\mathrm{x}) \wedge \mathrm{A}^{\prime}(\mathrm{x})\right)$ interpret $\forall \mathrm{xA}(\mathrm{x})$ and $\exists \mathrm{xA}(\mathrm{x})$ respectively, if $\mathrm{A}^{\prime}(\mathrm{x})$ interprets $\mathrm{A}(\mathrm{x})$.

${ }^{42}$ Kleene chose the coding $k=2^{n} \cdot 3^{m}$ so that $(k)_{0}=n$ and $(k)_{1}=m$ are the first two exponents in the prime factorization of $k$.
} 
relation " $e$ is the Gödel number of a partial recursive function which is defined at the argument $x$ with value $y$," abbreviated $\{e\}(x) \simeq y$.

Kleene associated with each arithmetical sentence $\mathrm{E}$ a relation $e$ realizes $\mathrm{E}$, defined inductively as follows:

1. $e$ realizes a prime sentence $\mathrm{s}=\mathrm{t}$ if $\mathrm{s}=\mathrm{t}$ is true under the intended interpretation.

2. $e$ realizes $\mathrm{A} \wedge \mathrm{B}$ if $(e)_{0}$ realizes $\mathrm{A}$ and $(e)_{1}$ realizes $\mathrm{B}$.

3. $e$ realizes $\mathrm{A} \vee \mathrm{B}$ if $(e)_{0}=0$ and $(e)_{1}$ realizes $\mathrm{A}$, or $(e)_{0}>0$ and $(e)_{1}$ realizes $\mathrm{B}$.

4. $e$ realizes $\mathrm{A} \supset \mathrm{B}$ if whenever $f$ realizes $\mathrm{A}$, then $\{e\}(f)$ is defined and realizes $\mathrm{B}$.

5. $e$ realizes $\neg \mathrm{A}$ if $e$ realizes $\mathrm{A} \supset 1=0$.

6. $e$ realizes $\forall \mathrm{xA}(\mathrm{x})$ if for every $n,\{e\}(n)$ is defined and realizes $\mathrm{A}(\mathbf{n})$.

7. $e$ realizes $\exists \mathrm{xA}(\mathrm{x})$ if $\left\{(e)_{1}\right\}(n)$ is defined and realizes $\mathrm{A}(\mathbf{n})$, where $n=(e)_{0}$.

The definition can be interpreted either constructively or classically. Kleene conjectured, and in [97] Kleene's student D. Nelson proved, that the universal closure of every axiom of intuitionistic arithmetic is realizable, and that if the universal closure(s) of the premise(s) of a rule of inference are realizable then so is the universal closure of the conclusion. Since $0=1$ is not realizable, it follows that every realizable sentence is consistent with HA. These results are known as "Nelson's Theorem:"

- Every arithmetical sentence which can be derived in intuitionistic arithmetic from realizable sentences is realizable.

- Every extension of intuitionistic arithmetic by realizable axioms is consistent.

Nelson's theorem established the consistency with HA of Church's Thesis $\left[\mathrm{CT}_{0}\right]$ in the form

$$
\forall \mathrm{x} \exists \mathrm{yA}(\mathrm{x}, \mathrm{y}) \supset \exists \mathrm{e} \forall \mathrm{x} \exists \mathrm{y}[\mathrm{T}(\mathrm{e}, \mathrm{x}, \mathrm{y}) \wedge \forall \mathrm{z}(\mathrm{U}(\mathrm{y}, \mathrm{z}) \supset \mathrm{A}(\mathrm{x}, \mathrm{z}))] .
$$

where $\mathrm{A}(\mathrm{x}, \mathrm{y})$ may be any formula containing only $\mathrm{x}$ and $\mathrm{y}$ free, and $\mathrm{T}(\mathrm{e}, \mathrm{x}, \mathrm{y})$ and $\mathrm{U}(\mathrm{y}, \mathrm{z})$ are decidable formulas expressing the primitive recursive relations " $y$ is the least gödel number of a computation of the value the partial recursive function with gödel number $e$ takes on the argument $x$ " and " $z$ is the value computed by the computation with gödel number $y$ " respectively. This schema, which is inconsistent with classical arithmetic, holds for the recursive mathematics of A. A. Markov. ${ }^{43}$ Markov's Principle $[\mathrm{MP}]$ is also realizable, by a proof using Markov's Principle informally. The consistency of $\mathbf{H A}+\mathrm{CT}_{0}+\mathrm{MP}$, expressing a large part of Russian recursive mathematics, follows.

Nelson formalized the notion "e realizes E" within a conservative extension $\mathbf{H A}$ ' of Heyting arithmetic with a new symbol $\mathbf{r}$, and proved that for all formulas A, B of the language of $\mathbf{H A}^{\prime}$ :

- If $\mathrm{A}, \mathrm{B}$ are sentences and $\mathrm{A} \supset \mathrm{B}$ is provable in $\mathbf{H A}^{\prime}$, so is $\mathbf{r A} \supset \mathbf{r B}$.

- The universal closure $\mathrm{C}$ of the formula $(A \supset \mathbf{r} A) \wedge(\mathbf{r} A \supset A)$ is realizable.

\footnotetext{
${ }^{43}$ Church's Thesis was not accepted by Brouwer or Heyting, but this formulation plays a parallel role for recursive mathematics to Brouwer's continuity principle for intuitionistic analysis, to be discussed in a later section.
} 
- The sentence $\mathbf{r} \mathrm{C}$ is provable in $\mathbf{H A}^{\prime}$.

Kleene [68] discussed, interpreted and extended Nelson's results. An important modification of what is now known as "number-realizability" was the relation e realizes- $\vdash \mathrm{E}$, obtained by changing the inductive cases for $\vee, \supset$ and $\exists$ as follows:

$3^{\prime} . e$ realizes $-\vdash \mathrm{A} \vee \mathrm{B}$ if $(e)_{0}=0$ and $(e)_{1}$ realizes $-\vdash \mathrm{A}$ and $\vdash \mathrm{A}$, or $(e)_{0}>0$ and $(e)_{1}$ realizes $-\vdash \mathrm{B}$ and $\vdash \mathrm{B}$.

$4^{\prime} . e$ realizes $-\vdash \mathrm{A} \supset \mathrm{B}$ if whenever $f$ realizes $-\vdash \mathrm{A}$ and $\vdash \mathrm{A}$, then $\{e\}(f)$ is defined and realizes $-\vdash \mathrm{B}$.

$7^{\prime} . e$ realizes $-\vdash \exists \mathrm{xA}(\mathrm{x})$ if $\left\{(e)_{1}\right\}(n)$ is defined and realizes $-\vdash \mathrm{A}(\mathbf{n})$ and $\vdash \mathrm{A}(\mathbf{n})$, where $n=(e)_{0}$.

The analogue of Nelson's theorem holds for realizability $-\vdash$. A more general result for realizability - $(\Gamma \vdash)$ (replacing " $\vdash$ " by " $\Gamma \vdash$ " in each inductive clause) is

- Every sentence which can be derived in intuitionistic arithmetic from realizable - $\vdash$ sentences $\Gamma$ is realizable- $(\Gamma \vdash)$.

Realizability - $\vdash$ gives the disjunction and existence properties for $\mathbf{H A}$, and the closure of HA under Church's Rule: If $\forall x \exists y A(x, y)$ is a closed theorem, so is $\forall x \exists y[T(\mathbf{e}, x, y) \wedge$ $\forall z(U(y, z) \supset A(x, z))]$ for some numeral e. It follows that HA can prove the existence only of provably recursive functions. ${ }^{44}$

Kleene initially hoped that realizability might provide a suitable notion of truth in intuitionistic arithmetic. Nelson's work verified that the schema

$$
(\mathrm{A} \supset \mathbf{r A}) \wedge(\mathbf{r A} \supset \mathrm{A})
$$

was consistent with HA. In [111], by carefully analyzing the inductive definition, Troelstra isolated a schema he called "extended Church's Thesis" $\left[\mathrm{ECT}_{0}\right]$ which had the effect over HA of identifying truth with realizability. Troelstra argued further that $\mathbf{H A}+\mathrm{ECT}_{0}+$ MP accurately represented the practice of Russian recursive mathematics.

By that time Kleene had found that extending his notion smoothly to intuitionistic analysis required the use of functions, not numbers, as realizing objects, and that this altered the class of realizable arithmetical formulas. Many modifications of realizability have since been developed as tools to obtain consistency and independence results for formal systems based on intuitionistic logic. Troelstra's [117] and van Oosten's [126], taken together, tell the most important parts of this intriguing story and bring it up to date.

\subsection{Kripke Semantics for IPC and IQC}

The completeness question for intuitionistic logic is subtle because IPC does not admit a finite truth-table interpretation (Gödel [50]) and every classical model, in the usual sense, of IQC is also a model of classical predicate logic. E. W. Beth [3], [4] and S. Kripke [83] each developed a "possible-world" semantics based on allowing decisions about truth or falsity of atomic sentences to be postponed from one moment in time to a later moment, and to be made in different ways along different branches in a partial ordering of possible

\footnotetext{
${ }^{44}$ In contrast, PA proves $\forall \mathrm{x} \exists \mathrm{y}[(\mathrm{y}=0 \wedge \mathrm{A}(\mathrm{x})) \vee(\mathrm{y}=1 \wedge \neg \mathrm{A}(\mathrm{x}))]$ for every arithmetical formula $\mathrm{A}(\mathrm{x})$.
} 
situations. Each proved a completeness theorem for IQC. Kripke's models are simpler than Beth's so we present them first, out of historical order. ${ }^{45}$

A Kripke model for intuitionistic propositional logic is a triple $\mathcal{K}=\{K, \leq, \Vdash\}$ where $K$ is an inhabited (i.e. effectively nonempty) set of nodes partially ordered by $\leq$, and the forcing relation $\Vdash$ is a binary relation between nodes and prime formulas $\mathrm{P}$ satisfying the restriction that if $k, k^{\prime} \in K$ and $k \leq k^{\prime}$ and $k \Vdash \mathrm{P}$ then also $k^{\prime} \Vdash \mathrm{P}$. ${ }^{46}$ The forcing relation is extended inductively to all formulas as follows:

1. $k \Vdash \mathrm{A} \wedge \mathrm{B}$ if $k \Vdash \mathrm{A}$ and $k \Vdash \mathrm{B}$.

$2 . k \Vdash \mathrm{A} \vee \mathrm{B}$ if $k \Vdash \mathrm{A}$ or $k \Vdash \mathrm{B}$.

3. $k \Vdash \mathrm{A} \supset \mathrm{B}$ if, for each $k^{\prime} \geq k$ : if $k^{\prime} \Vdash \mathrm{A}$ then $k^{\prime} \Vdash \mathrm{B}$.

4. $k \Vdash \neg \mathrm{A}$ if there is no $k^{\prime} \geq k$ such that $k^{\prime} \Vdash \mathrm{A}$.

It is straightforward to prove that the forcing relation is monotone, consistent, and sound for IPC:

- For every formula $\mathrm{E}$ : If $k \leq k^{\prime}$ and $k \Vdash \mathrm{E}$ then $k^{\prime} \Vdash \mathrm{E}$.

- For no $k \in K$ and no formula $\mathrm{E}$ do both $k \Vdash \mathrm{E}$ and $k \Vdash \neg \mathrm{E}$ hold.

- If $\vdash \mathrm{E}$ then $k \Vdash \mathrm{E}$ for every $k \in K$.

A Kripke model is a tree model if each node $k$ has a unique immediate predecessor in the ordering $\leq$ of $K$. The tree is rooted if there is a (necessarily unique) $k_{0} \in K$ such that $k_{0} \leq k$ for every $k \in K$. The strong completeness result, from which the decidability of intuitionistic propositional logic follows, is

- To each formula $\mathrm{E}$ which is not a theorem of IPC there is a finite rooted tree Kripke model whose root $k_{0}$ does not force $\mathrm{E}{ }^{47}$

For example, a simple Kripke counterexample to $\neg \mathrm{P} \vee \neg \neg \mathrm{P}$ is given by $K=\left\{k_{0}, k_{1}, k_{2}\right\}$ where $k_{0}<k_{1}, k_{0}<k_{2}$ and $k_{1}, k_{2}$ are incomparable, with the forcing relation determined by $k_{1} \Vdash \mathrm{P}$.

A Kripke model for intuitionistic predicate logic is a quadruple $\mathcal{K}=\{K, \leq, D \Vdash\}$ where $K$ is an inhabited set of nodes partially ordered by $\leq$, and for each $k \in K$ the domain of $k$ is an inhabited set $D(k)$ of objects with the restriction that if $k, k^{\prime} \in K$ and $k \leq k^{\prime}$ then $D(k) \subseteq D\left(k^{\prime}\right)$. The forcing relation can be thought of as a binary relation between nodes and prime sentences of an expanded language with an individual constant for each element of $\bigcup_{k \in \mathcal{K}} D(k)$, with the restriction that if $k \in K$ and $k \Vdash \mathrm{P}$ where $\mathrm{P}$ is prime, then every constant occurring in $\mathrm{P}$ is an element of $D(k)$ and if $k^{\prime} \in K$ with $k \leq k^{\prime}$ then also $k^{\prime} \Vdash \mathrm{P} .{ }^{48}$ The forcing relation extends to all sentences of the expanded language using, in addition to the clauses for the propositional connectives, the following:

\footnotetext{
${ }^{45}$ Kripke observed that his models can easily be transformed into Beth models of a special kind. His completeness proof was based on Beth's, which will be described in a later section.

${ }^{46}$ The "forcing" terminology was not used in this way by Kripke but is now standard. In Kripke's original definition each prime formula $\mathrm{P}$ was assigned a truth value $\mathrm{T}$ or $\mathrm{F}$ at each node $k$, with the condition that if $k \leq k^{\prime}$ and $\mathrm{P}$ was $\mathrm{T}$ at $k$ then also $\mathrm{P}$ was $\mathrm{T}$ at $k^{\prime}$. Here " $k \Vdash \mathrm{A}$ " corresponds to "A is $\mathrm{T}$ at $k$."

${ }^{47}$ For details see [118] or [108], where the Kripke completeness theorems for IPC and IQC are presented using the method of Aczel [1].

${ }^{48}$ Instead of expanding the language, Kripke associated with each node $k$ and each $n$-place prime formula $\mathrm{P}$ a subset of $[D(k)]^{n}$ on which $\mathrm{P}$ was $\mathrm{T}$ at $k$, with the corresponding monotonicity requirement. The two approaches are equivalent classically, and for counterexamples it is convenient to have names in the language for elements of the domain.
} 
5. $k \Vdash \forall \mathrm{xA}(\mathrm{x})$ if, for each $k^{\prime} \geq k$ and each $d^{\prime} \in D\left(k^{\prime}\right): k^{\prime} \Vdash \mathrm{A}\left(\mathrm{d}^{\prime}\right)$.

6. $k \Vdash \exists \mathrm{xA}(\mathrm{x})$ if, for some $d \in D(k): k \Vdash \mathrm{A}(\mathrm{d})$.

Monotonicity, consistency and soundness extend naturally to the predicate case. The completeness theorem takes the form

- If $\mathrm{E}$ is a sentence of the language of predicate logic which is forced at every node of every Kripke model, then E is provable in IQC.

The argument actually shows that the assumption that $\mathrm{E}$ is unprovable is contradictory; the existence of a proof then follows by informal Markov's Principle. Following Gödel, Kreisel [80] argued that completeness of intuitionistic predicate logic entails Markov's Principle, but Veldman [128] found a way to escape this conclusion by modifying Kripke's definition to allow "exploding" nodes at which all sentences of the relevant language are forced. The modification involved adding a propositional constant $\perp$ for absurdity to the language and defining $\neg \mathrm{A}$ to be $\mathrm{A} \supset \perp$, so clause 4 of the definition could be omitted. Because $\perp$ was a new prime sentence, $k \Vdash \perp$ could happen at one or more nodes $k$ of a Veldman model. Because $\vdash \neg \perp$ (because $\vdash \perp \supset \perp$ ), if $k \Vdash \perp$ then $k \Vdash \mathrm{E}$ for every sentence E.

From the classical point of view, a Kripke model is just a Veldman model with all the exploding nodes omitted. But Veldman's intuitionistic completeness proof is a beautiful application of Brouwer's Fan Theorem, a principle of intuitionistic analysis. Since Beth's completeness proof is best understood in terms of the intuitionistic notion of spread, it is time for us to explore Brouwer's boldest creation.

\section{The Second Act of Intuitionism}

From the beginning Brouwer struggled with the problem of the continuum. In his dissertation, while rejecting Kant's claim for an a priori intuition of space, Brouwer admitted the need for a separate intuition of the continuum:

...the basic intuition of mathematics (and of any intellectual activity) as the substratum, divested of all quality, of any perception of change, a unity of continuity and discreteness, a possibility of thinking together several entities, connected by a 'between', which is never exhausted by the insertion of new entities. ... Having recognized that the intuition of continuity of 'fluidity', is as primitive as that of several things conceived as forming together a unit, the latter being at the basis of every mathematical construction, we are able to state properties of the continuum as a 'matrix of points to be thought of as a whole'. ([10] p. 17.)

Then, after showing how to construct from the integers the rational numbers with their dense linear ordering (of order type $\eta$ ), he affirmed a form of completeness with the following justification:

The mathematical intuition is unable to create other than denumerable sets of individuals. But it is able, after having created a scale of ordertype $\eta$, to superimpose upon it a continuum as a whole, which afterwards can be taken conversely as a measurable continuum, which is the matrix of the points on the scale. ([10] p. 45.) 
The problem was to reconcile this independent intuition of the continuum with the fact that the only real numbers Brouwer then considered to be legitimate mathematical entities were the definable real numbers. He believed that only finite or denumerably infinite sets could be constructed, and he accepted Cantor's diagonal argument for the nondenumerability of the continuum. Moreover, every denumerable set must have measure zero, while the continuum should have positive measure. ${ }^{49}$ This conflict led the young Brouwer to the theoretical notion of a "denumerably unfinished set" to describe such aggregates as Cantor's second number class, "the totality of definable points on the continuum, and a fortiori the totality of all possible mathematical systems" ([10] p. 82). Such sets, which would have to consist of more than denumerably many individual elements, could not exist mathematically and so could not be put in one-to-one correspondence with the continuum.

From the foundational viewpoint Brouwer was not satisfied with this ad hoc solution. In [14] and [15] he finally succeeded in developing an intuitionistic structure for the continuum, or more precisely its underlying Baire space consisting of the infinitely proceeding sequences of natural numbers with the initial-segment topology. His construction could be interpreted classically or intuitionistically; however, by considering the intuitionistically acceptable ways of proving properties of the entire structure, Brouwer was led to two new principles one of which contradicted the natural classical interpretation. With this bold step he established intuitionistic analysis as an alternative to classical analysis, not just a constructive part thereof.

\subsection{Species, Spreads and Infinitely Proceeding Sequences}

In 1918 and 1919 Brouwer published two fundamental papers with the title "Foundation of set theory independent of the logical principle of the excluded middle" (Begründung der Mengenlehre unabhängig vom logischen Satz vom ausgeschlossenen Dritten). The first ([14]) had the subtitle "General set theory" (Allgemeine Mengenlehre) and the second ([15]) "Theory of sets of points" (Theorie der Punktmengen). He began by distinguishing between the notions Species and Menge. A structured set (Menge) was a mathematical entity of a definite kind, while a species of first order (Spezies erster Ordnung) was "a property which only a mathematical entity can possess, in which case it [the entity] is called an element of the species of first order..." ([14] p. 4). A species of second order was a property which could be possessed by species of first order, and so on.

Brouwer explicitly rejected the comprehension axiom of classical set theory, according to which each property determined a set (or a subset of a given set). Instead, he accepted sets of choice sequences, infinitely proceeding sequences of mathematical entities previously constructed, where each successive element was allowed to be chosen more or less freely, restricted only by the finitely many choices already made and by the law determining the set. The English term eventually chosen by Brouwer ([28]) for this kind of structured set was "spread." Here is the definition he gave in $[14]:^{50}$

A spread is a law on the basis of which, when again and again an arbitrary integer (Ziffernkomplex) is chosen from the sequence $\zeta$ [of the positive integers $1,2,3, \ldots]$, each of these choices produces either a particular symbol (Zeichen), or nothing, or else leads to the termination (Hemmung) of the process and the

\footnotetext{
${ }^{49}$ Sixty years later Errett Bishop [8] succeeded in developing a constructive measure theory on a continuum consisting only of constructive real numbers, making implicit use of the fact that these numbers cannot be enumerated constructively.

${ }^{50}$ The translation from the German is mine.
} 
definitive annihilation of its results, where for each $n$ after an unterminated sequence of $n-1$ choices at least one number can be specified which, if it is chosen as the $n$th number, does not lead to the termination of the process. Every sequence (which therefore in general is not representable as finished) of symbols chosen in this way from the spread is called an element of the spread. We will also denote the common manner of generation of the elements of a spread $M$ briefly by the spread $M .{ }^{51}$

The spread law is twofold, consisting of a choice law determining which integers are available to be chosen at each stage of the process, and a correlation law determining which symbol is attached to each permitted finite sequence of choices. Brouwer left open the interpretation of "law" in this definition, and it is consistent to assume that every choice sequence determines a spread of which it is the only element. ${ }^{52}$ Certainly a spread law may be such that after a particular sequence of $n-1$ permitted choices, every subsequent choice is determined in advance and the process never terminates.

In his popular but detailed exposition "Intuitionism: An Introduction" ([60]) Heyting simplified the definition of spread by omitting the possibility of termination (which could be indicated instead by assigning a special symbol and requiring all future choices to be 1, for example), and wrote:

... by an infinitely proceeding sequence (abbreviated: ips) we mean exactly what the words express, i.e. a sequence that can be continued ad infinitum. The question how the components of the sequence are successively determined, whether by a law, by free choices, by throwing a die, or by some other means, is entirely irrelevant.

Infinitely proceeding sequences whose components were completely determined by a (finite) definition were called by Brouwer "fundamental sequences" or "sharp arrows." Other authors have called them "lawlike." Kreisel ([81]) introduced a complementary notion of "lawless" or "absolutely free" choice sequence, obeying the second-order restriction that no restriction would ever be imposed on the choices allowed. ${ }^{53}$

A fan or finitary spread is one with the property that after each sequence $a_{1}, \ldots, a_{n-1}$ of successive choices permitted by the choice law, only finitely many integers (how many and which ones depending on the choices already made) are available to be chosen as the value of $a_{n}$. A fan is the intuitionistic analogue of a compact set in classical mathematics. At the other extreme is the universal spread, in which every natural number is available to be chosen at each stage of the construction, and the symbol correlated after each successive choice is the numeral for the number just chosen.

Brouwer was especially interested in the spread of real number generators, each representing (in one version) an infinitely proceeding sequence of nested closed intervals with rational endpoints and lengths converging toward zero, or (in another) a Cauchy sequence $\left\{r_{n}\right\}$ of rational numbers. ${ }^{54}$ In particular, a canonical real number generator can be defined (cf. [60] p. 41) as an ips $\left\{x_{n} 2^{-n}\right\}$, where the choice law determining which sequences

\footnotetext{
${ }^{51}[14]$ p. 4. In his introduction to Part I of [87] van Stigt points out that the idea of a structured set was already present in Brouwer's 1914 review of a book by Schoenflies and Hahn. The same volume contains van Stigt's English translation of Brouwer's 1921 Dutch summary of [14] and [15], including a paragraph similar to this.

${ }^{52}$ This relative interpretation is implicit in Kleene and Vesley's [74].

${ }^{53}$ In [82] Kreisel and Troelstra developed a formal theory of choice sequences based on these two concepts.

${ }^{54}\left\{r_{n}\right\}$ is Cauchy if for every positive integer $k$ there is an $n$ such that for all $p:\left|r_{n}-r_{n+p}\right|<1 / k$.
} 
$\left\{x_{n}\right\}$ of integers may be chosen satisfies

$$
\left|x_{n} 2^{-n}-x_{n+1} 2^{-n-1}\right| \leq 2^{-n-1}
$$

or equivalently $\left|2 x_{n}-x_{n+1}\right| \leq 1$. The finite initial segments of such a sequence would give better and better rational approximations to the real number in the process of generation, and this was the only information one could expect to have about an arbitrary real number generator. Thus the general question whether any two real number generators coincide, or converge to the same limit, is intuitionistically undecidable. ${ }^{55}$

By asserting that spreads and their elements were legitimate mathematical entities, Brouwer accepted infinitely proceeding sequences of natural numbers, even though "most" such sequences had no possibility of ever being completely defined. By accepting species of every finite order he made it possible to compare the intuitionistic theory of properties with classical abstract set theory. This broadening of the concept of a legitimate mathematical entity, with the new insights it engendered, was called by Brouwer "the second act of intuitionism."

\subsection{Brouwer's Fan Theorem, Continuity Principle, and Bar Theorem}

Brouwer was not an easy expositor of his own ideas and he published mostly in Dutch or German. The first edition [60] of Heyting's delightful "Intuitionism: An Introduction" appeared in 1956, clarifying the intuitionistic approach to real numbers. After describing in detail the arithmetic of real number generators, Heyting proved that every real number generator (abbreviated r.n.g.) coincides with a canonical r.n.g. (abbreviated c.r.n.g.). The choice law for the spread of the c.r.n.g. allows any integer to be chosen as $x_{1}$ and from then on requires $x_{n+1} \in\left\{2 x_{n}-1,2 x_{n}, 2 x_{n}+1\right\}$, so except for the first choice it is finitary.

If $x=\left\{x_{n} 2^{-n}\right\}$ and $y=\left\{y_{n} 2^{-n}\right\}$ are c.r.n.g., then $x<y$ if and only if for some $n: x_{n}+2<y_{n}$, and then $y_{n+p} 2^{-(n+p)}-x_{n+p} 2^{-(n+p)}>2^{-n}$ for all $p$. If $a$ and $b$ are c.r.n.g., the closed interval $[a, b]$ consists of all c.r.n.g. $x, y$ such that $\neg(x<a \wedge y<b)$ and $\neg(x>a \wedge y>b)$. The basic representation theorem, stated and proved in [60] p. 42, where it is attributed to Brouwer ([15] and [19]), is then

Theorem 1. Every closed interval of the continuum coincides with a finitary spread [of canonical real number generators]. ${ }^{56}$

Next Heyting stated and proved "Brouwer's Fan Theorem" in a form which is not strictly true classically but which illustrates the intuitionistic concept of a total function: ${ }^{57}$

\footnotetext{
${ }^{55}$ Two real number generators $r \equiv\left\{r_{n}\right\}$ and $s \equiv\left\{s_{n}\right\}$ coincide (notation: $r=s$ ) if for every positive integer $k$ there is an $n$ such that for all $p:\left|r_{n+p}-s_{n+p}\right|<1 / k$. If, on the other hand, there is a $k$ so that for all $p: r_{n+p}>s_{n+p}+1 / k$, then $r>s$ (and $s<r$ ). The classical trichotomy law $r<s \vee r=s \vee r>s$ fails intuitionistically, but the following useful substitute holds: If $r<s$ then $r<t \vee t<s$ for every real number generator $t([60]$ p. 25]). Apartness of real number generators is a positive notion; precisely, $r$ is apart from $s$ if there exist $k$ and $n$ such that for all $p:\left|r_{n+p}-s_{n+p}\right|>1 / k$. It follows that $r$ is apart from $s$ just in case $r<s \vee r>s$, so if $r$ is not apart from $s$ then $r$ and $s$ coincide. This result appeared in [Brouwer, 1930], the published version of a lecture Brouwer gave in Vienna in March 1928, with Gödel in the audience.

${ }^{56}$ There is a subtlety here, as each rational number of the form $m 2^{-n}$ is the limit of two distinct c.r.n.g. one approaching it from the left and one from the right; and these two approximating sequences coincide.

${ }^{57}$ In mathematical terms, these two theorems together assert that every function defined everywhere on a closed bounded interval is uniformly continuous. Classically it is true that every continuous total function on a closed bounded interval is uniformly continuous. Brouwer's intuition led him to the additional assumption that every (calculable) total function is continuous.
} 
Theorem [2]. If an integer-valued function $\varphi(\delta)$ is defined for every element $\delta$ of a finitary spread $S$, then a natural number $[N]$ can be computed from the definition of $\varphi$, such that $\varphi(\delta)$ is determined by the first $N$ components of $\delta$; that is, if $\delta_{1}$ and $\delta_{2}$ are such elements of $S$ that the first $N$ components of $\delta_{1}$ are equal to the first $N$ components of $\delta_{2}$, then $\varphi\left(\delta_{1}\right)=\varphi\left(\delta_{2}\right)$.

As references Heyting cited Brouwer's [18], [19], [22], [26] and [27]. In the proof he worked with the underlying fan $K$ of infinitely proceeding sequences $d$ of integers allowed by the choice law of $S$, and with the function $f(d)=\varphi(\delta)$ where $\delta$ is the element of $S$ associated with $d$ by the correlation law.

First he observed that "As $f(d)$ must be calculable, its value must be determined by a finite number of the components of $d$; that is to say, by a sequence $a(d)$ in $F$ " (where $F$ is the species of finite sequences of integers which are permitted by the choice law). This is an instance of Brouwer's continuity principle (discussed below). Then Heyting continued: "Let $C$ be the species of the $a(d)$ which correspond in this way to the elements of $K$; then every element of $K$ has a segment $a(d)$ in $C$. Also, if $b$ is a sequence in $F$, every $K$-continuation of $b$ has a segment in $C$; we shall express this property by saying that $b$ is $K$-barred by $C . "$

At this point a classical mathematician would invoke König's Lemma, which says in this context that if a fan has unbarred finite branches of arbitrary length then it has an infinite unbarred branch, to conclude by reductio ad absurdum that there is an $N$ such that every $b$ in $F$ has a segment in $C$ of length $\leq N$. But the use of proof by contradiction to establish a positive conclusion (here, the existence of $N$ ) is not allowed intuitionistically. Instead, Heyting invoked Brouwer's "Bar Theorem" (explained below) to conclude that such an $N$ exists, completing the proof.

The heart of Brouwer's reconstruction of analysis was contained in his continuity principle and his principle of bar induction. The continuity principle depended on a strictly finitary interpretation of the concept of a total function on a spread, according to which the value such a function takes on an arbitrary element $\delta$ of the spread must be determined on the basis of a finite initial segment of the underlying i.p.s. $d$ to which $\delta$ is correlated. This conflicted with the classical existence of discontinuous functions, for example the function defined on all infinite sequences of $0 \mathrm{~s}$ and $1 \mathrm{~s}$ which takes the value 0 on the identically zero sequence, and 1 on every sequence which is not identically zero, since any finite sequence of $0 \mathrm{~s}$ can be followed by a 1 and then continued indefinitely. In short, the continuity principle provided a proof of

$$
\neg \forall \delta(\forall x \delta(x)=0 \vee \neg \forall x \delta(x)=0),
$$

giving a strong counterexample to the classical law of excluded middle.

The principle of bar induction (which Brouwer called the "Bar Theorem") for the universal spread of all choice sequences of natural numbers can be stated as follows. If to each i.p.s. $\alpha$ of natural numbers a natural number $n_{\alpha}$ can be calculated such that the finite sequence $\left\langle\alpha(1), \ldots, \alpha\left(n_{\alpha}\right)\right\rangle$ belongs to the species $C$ (called a bar for the universal spread), and if each element of $C$ is also an element of the species $A$, where $A$ has the property that if $\left\langle a_{1}, \ldots, a_{n-1}, k\right\rangle \in A$ for every $k$ then also $\left\langle a_{1}, \ldots, a_{n-1}\right\rangle \in A$, then the empty sequence \langle\rangle belongs to $A$. The principle follows for every spread, in particular for every fan. Brouwer gave a proof of the principle based on an analysis of the types of inference which might be used to prove properties $A$ of choice sequences. In the first edition of [60] Heyting repeated this argument, but in the second edition (published in 1966) he accepted Kleene's conclusion that the "Bar Theorem" was actually a new assumption. 


\subsection{Kleene and Vesley's Formalism for Intuitionistic Mathematics}

Even before Heyting's [60] appeared, Kleene had been working on an axiomatization of intuitionistic analysis with the goal of making Brouwer's reasoning comprehensible and accessible, especially to classical mathematicians. In 1965 Kleene and Vesley published "The Foundations of Intuitionistic Mathematics, Especially in Relation to Recursive Function Theory" containing a complete axiomatization of the theory of infinitely proceeding sequences including real number generators. The formal system FIM was based on a twosorted language, with variables $x, y, \ldots$ for numbers and $\alpha, \beta, \ldots$ for choice sequences. Its mathematical axioms included those of Heyting arithmetic (with additional primitive recursive functions), an axiom schema of countable choice (or alternatively a comprehension schema asserting the existence of definable functions), a strong axiom schema of continuous choice which Kleene called "Brouwer's Principle for Functions," and an axiom schema of bar induction which Kleene showed to be independent of the other axioms.

Vesley worked out the application to real number generators. Using functions rather than numbers as realizing objects, Kleene succeeded in giving a consistency proof for FIM relative to its classically correct subsystem B (without Brouwer's Principle). Although FIM is inconsistent with the classical law of excluded middle, Kleene's realizability interpretation and Vesley's thorough development of the theory of real number generators justified Brouwerian analysis as a coherent alternative to classical analysis.

Brouwer's Principle made it possible to prove strong undecidability results within intuitionistic analysis, obviating the need for weak counterexamples such as the one Heyting used to show the unprovability, in his predicate logic, of $(\mathrm{x}) \neg \neg \mathrm{a} \supset \neg \neg(\mathrm{x}) \mathrm{a}$. If $\mathrm{A}(\alpha, \mathrm{x})$ is the formula $\alpha(x)=0$ then the following are formal theorems of FIM which contradict classical logic:

$$
\begin{aligned}
& \text { - } \vdash \neg \forall \alpha[\forall \mathrm{xA}(\alpha, \mathrm{x}) \vee \neg \forall \mathrm{xA}(\alpha, \mathrm{x})] \quad\left({ }^{*} 27.17\right) . \\
& \text { - } \vdash \neg \forall \alpha[\neg \exists \mathrm{x} \neg \mathrm{A}(\alpha, \mathrm{x}) \vee \neg \neg \exists \mathrm{x} \neg \mathrm{A}(\alpha, \mathrm{x})] \quad\left({ }^{*} 27.18\right) . \\
& \text { - } \vdash \neg \forall \alpha[\forall \mathrm{x}(\mathrm{A}(\alpha, \mathrm{x}) \vee \neg \forall \mathrm{xA}(\alpha, \mathrm{x})) \supset \forall \mathrm{xA}(\alpha, \mathrm{x}) \vee \neg \forall \mathrm{xA}(\alpha, \mathrm{x})] \quad\left({ }^{*} 27.19\right) .
\end{aligned}
$$

If $\mathrm{B}(\alpha)$ is $\forall \mathrm{xA}(\alpha, \mathrm{x}) \vee \neg \forall \mathrm{xA}(\alpha, \mathrm{x})$ then $\vdash \forall \alpha \neg \neg \mathrm{B}(\alpha)$ (cf. formal theorem 4.8 in Heyting's propositional logic) and so $\vdash \neg[\forall \alpha \neg \neg \mathrm{B}(\alpha) \supset \neg \neg \forall \alpha \mathrm{B}(\alpha)]$ by ${ }^{*} 27.17$, giving a strong counterexample to double negation shift in place of Heyting's weak one. Here, of course, we are implicitly using the consistency of FIM.

Realizability and its variants provide relative consistency and independence proofs for a variety of principles which have been proposed to extend intuitionistic analysis. A strong version $\forall \alpha[\neg \forall \mathrm{x} \neg \mathrm{A}(\alpha, \mathrm{x}) \supset \exists \mathrm{xA}(\alpha, \mathrm{x})]$ of Markov's Principle, while realizable classically and hence consistent with FIM, is unprovable (Kleene [74] p. 131, using a modification of function-realizability inspired by Kreisel [79]). A weak form $\forall \alpha \neg \neg \mathrm{GR}(\alpha)$ of Church's Thesis is also consistent with and independent of FIM, where $\operatorname{GR}(\alpha)$ is a formula of the form $\exists \mathrm{e} \forall \mathrm{x} \exists \mathrm{y}[\mathrm{T}(\mathrm{e}, \mathrm{x}, \mathrm{y}) \wedge \forall \mathrm{z}(\mathrm{U}(\mathrm{y}, \mathrm{z}) \supset \alpha(\mathrm{x})=\mathrm{z})]$ expressing " $\alpha$ is general recursive" (J. R. Moschovakis [91], using a modified function-realizability interpretation inspired by Vesley [129]). The strong form of Church's Thesis conflicts with Brouwer's Principle, and in the presence of Markov's Principle the weak form conflicts with the Bar Theorem (cf. $[93])$.

Troelstra [112] proposed an extension GC (for "generalized continuity") of Brouwer's Principle, and used it to characterize function-realizability just as he had used $\mathrm{ECT}_{0}$ to axiomatize number-realizability. On the other hand, beginning with the observation 
that FIM is consistent with classical arithmetic, Kleene [71] surveyed the possibilities of extending intuitionistic analysis consistently in the classical direction. For other examples see [117] and [126].

\subsection{Heyting's Arithmetic of Species and HA ${ }^{\omega}$}

In [60] (p. 37) Heyting repeated Brouwer's definition from [14] ("Definition 1") of a species as "a property which mathematical entities can be supposed to possess," and added:

Definition 2. After a species $\mathcal{S}$ has been defined, any mathematical entity which has been or might have been defined before $\mathcal{S}$ and which satisfies the condition $\mathcal{S}$, is a member of the species $\mathcal{S}$.

The "or might have been" allows for the possibility of infinite species of natural numbers or choice sequences. Heyting gives examples: "the property [of a real number generator] of coinciding with a given number-generator is a species," and "The components of an ips $\xi$ of natural numbers form a species ...." These are species of type 0 , while the continuum (consisting of species of type 0 , as in the first example) is a species of type 1 .

If $S$ and $T$ are species such that every element of $T$ is also an element of $S$, then $T$ is a subspecies of $S(T \subseteq S)$, and $S-T$ is the subspecies of those elements of $S$ which cannot belong to $T$. Two species $S$ and $T$ are equal if $S \subseteq T$ and $T \subseteq S$ (so equality of species is extensional). "If $T \subseteq S$ then $S^{\prime}=T \cup(S-T)$ is not always identical with $S$, for $S^{\prime}$ contains only those elements of $S$ for which it can be decided whether they belong to $T$ or not." However, $S^{\prime}$ is congruent to $S$ (in the sense that neither species can contain an element which does not belong to the other). If $S^{\prime}=S$ then $T$ is a detachable subspecies of $S$. There are many detachable subspecies of the natural numbers, but "the continuum has no other detachable subspecies than itself and the null species."

Troelstra [112] expanded on Heyting's presentation in [60] of the intuitionistic theory of species, providing a formal system $\mathbf{H A} \mathbf{S}_{0}$ extending $\mathbf{H A}$ with variables for numbers and species of numbers, formulating axioms EXT of extensionality and ACA of arithmetical comprehension, and proving that $\mathbf{H A S}_{0}+\mathrm{ACA}+\mathrm{EXT}$ is conservative over HA. In [113] he stated and proved the uniformity principle $[\mathrm{UP}]$ :

$$
\forall \mathrm{X} \exists \mathrm{n} \mathrm{A}(\mathrm{X}, \mathrm{n}) \supset \exists \mathrm{n} \forall \mathrm{XA}(\mathrm{X}, \mathrm{n}),
$$

explicitly contradicting classical second-order logic. A similar principle $\left[\mathrm{UP}_{1}\right]$ holds for

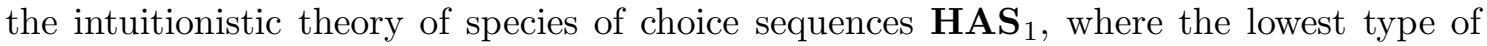
variable ranges over infinitely proceeding sequences of natural numbers. The uniformity principle implies that the only detachable subspecies of an arbitrary species of type 1 are the species itself and the null species, generalizing Heyting's comment quoted above.

Another extension of HA explored by Troelstra and others is intuitionistic arithmetic of arbitrary finite type $\mathbf{H A}^{\omega}$ (called $\mathbf{N}-\mathbf{H A}^{\omega}$ in [112]), with variables of type 0 (over natural numbers) and for each pair $\sigma, \tau$ of types also variables of type $(\sigma, \tau)$ (over functions from objects of type $\sigma$ to objects of type $\tau$ ). Equality between objects of the same type may be taken to be intensional, hence decidable (in $\mathbf{I}-\mathbf{H A}^{\omega}$, where $=$ expresses identity) or extensional (in $\mathbf{E}-\mathbf{H} \mathbf{A}^{\omega}$ ) as in Gödel's Dialectica interpretation and the theory of choice sequences. The axioms include those of first-order Heyting arithmetic with induction extended to arbitrary formulas of the language, and schemas defining the function constants of projection, application and primitive recursion at all appropriate types. ${ }^{58}$

\footnotetext{
${ }^{58}$ See the discussion in Beeson [6], a rich source of information about formalisms proposed before 1985 for theories based on intuitionistic logic, including intuitionistic set theory and Bishop's constructive math-
} 


\subsection{Beth Models and Veldman's Completeness Proof for IQC}

A Beth model for IQC is a quintuple $\mathcal{M}=\left\{B, \leq, k_{0}, D \Vdash\right\}$ where $(B, \leq)$ is a finitely branching tree with root $k_{0}$, the domain function $D$ maps each $k \in B$ to a set of positive integers where $D(k) \subseteq D\left(k^{\prime}\right)$ if $k \leq k^{\prime}$, and the forcing relation for prime sentences $\mathrm{P}$ (of the language of IQC enhanced by constants for the positive integers) satisfies the condition that $k \Vdash \mathrm{P}$ if and only if every branch through $k$ eventually encounters a node $k^{\prime}$ such that $k^{\prime} \Vdash \mathrm{P}$. Beth forcing extends inductively to compound formulas in the same way as Kripke forcing except in the cases of disjunction and existence, which are treated as follows:

2. $k \Vdash \mathrm{A} \vee \mathrm{B}$ if every branch through $k$ eventually hits a node $k^{\prime}$ such that $k^{\prime} \Vdash \mathrm{A}$ or $k^{\prime} \Vdash \mathrm{B}$.

6. $k \Vdash \exists \mathrm{xA}(\mathrm{x})$ if every branch through $k$ eventually hits a node $k^{\prime}$ such that, for some $d \in D\left(k^{\prime}\right): k^{\prime} \Vdash \mathrm{A}(\mathrm{d})$.

In effect, $k \Vdash \mathrm{A} \vee \mathrm{B}$ if the subtree through $k$ is barred by the disjoint union of two species $C$ and $D$, where each node in $C$ forces A and each node in $D$ forces B; and similarly for $\exists$. Beth ([4] p. 447) gave his definition in terms of validity on subtrees, so e.g. A $\vee \mathrm{B}$ is valid on $\mathcal{M}$ if $\mathcal{M}$ is the union of finitely many (compatible) subtrees on each of which either A or B is valid. Fan induction (bar induction on a finitary spread), or classical König's Lemma, establishes the equivalence of his definition with the one given here. ${ }^{59}$

Monotonicity and soundness are proved by the usual inductive arguments. The completeness theorem asserts that every sentence $\mathrm{E}$ which is unprovable in IQC has a Beth countermodel, i.e. a Beth model whose root does not force E. The proof consists of a systematic search for a proof of E, using a diagram called a Beth tableau to guide the construction of a finitely branching tree with sets of formulas attached to the nodes. If the tableau is closed, giving a proof of E, then every branch of the tree is finite; if not, then the resulting tree is a Beth countermodel to E.

Citing Dyson and Kreisel [38], Veldman ([128]) observed that Beth's construction was inadequate at least from the intuitionistic viewpoint, although Beth's ideas were used in Kripke's classical completeness proof. By modifying Kripke semantics to allow exploding nodes (see $\S 6.3$ for the definition), Veldman succeeded in giving an intuitionistic proof of "the existence of a universal modified Kripke-model, i.e. a model such that every sentence, true in the model, is derivable in intuitionistic predicate calculus" while every intuitionistically provable sentence is true in the model. A discussion of his method seems a suitable ending for this historical exposition of the logic of Brouwer and Heyting.

Aczel ([1]) used the notion of saturation to provide a perspicuous classical proof of the Kripke completeness of IQC. A set $\Gamma$ of sentences of the language $\mathcal{L}[C]$, the pure predicate language (with $\perp$ instead of $\neg$ ) expanded by a set $C$ of individual constants, is $C$-saturated if it satisfies the following conditions:

(i) $\Gamma$ has at least one element.

(ii) If $\Gamma \vdash \mathrm{A} \vee \mathrm{B}$ where $\mathrm{A} \vee \mathrm{B} \in \mathcal{L}[C]$, then $\mathrm{A} \in \Gamma$ or $\mathrm{B} \in \Gamma$.

(iii) If $\Gamma \vdash \exists \mathrm{xA}(\mathrm{x})$ where $\exists \mathrm{xA}(\mathrm{x}) \in \mathcal{L}[C]$, then $\mathrm{A}(\mathrm{c}) \in \Gamma$ for some constant $\mathrm{c} \in C$.

\footnotetext{
ematics. Troelstra's [115] discusses choice sequences informally from a variety of perspectives; cf. [82].

${ }^{59}$ In their detailed presentation, Troelstra and van Dalen ([118] p. 677ff.) observe that Beth used a constant domain function while van Dalen ([121]) allowed expanding domains.
} 
Condition (ii) guarantees that a $C$-saturated set is closed under deducibility, since if $\Gamma \vdash \mathrm{A}$ then also $\Gamma \vdash \mathrm{A} \vee \mathrm{A}$. For Veldman's proof we do not require that $\Gamma$ be consistent.

To construct the universal Veldman model we need a denumerably infinite set $C=$ $\left\{c_{i, k}\right\}$ of distinct individual constants, and an enumeration $\mathrm{A}_{0}, \mathrm{~A}_{1}, \mathrm{~A}_{2}, \ldots$ without repetition of the sentences of $\mathcal{L}[C]$ such that each $\mathrm{A}_{n}$ contains only constants $c_{i, k}$ with $i, k<n$, or equivalently with $j(i, k)<j(0, n)$ where $j$ is the surjective pairing function obtained from the enumeration

$$
\langle 0,0\rangle ;\langle 0,1\rangle,\langle 1,0\rangle,\langle 1,1\rangle ;\langle 0,2\rangle,\langle 1,2\rangle,\langle 2,0\rangle,\langle 2,1\rangle,\langle 2,2\rangle ;\langle 0,3\rangle, \ldots
$$

The simplest solution is to take $c_{i, k}=j(i, k)$, so $C=\{0,1,2, \ldots\}$. We need the corresponding projection functions $j_{0}, j_{1}$ so $j_{0}(j(i, k))=i, j_{1}(j(i, k))=k$ and $j\left(j_{0}(h), j_{1}(h)\right)=h$. We need an enumeration $d_{0}, d_{1}, \ldots$ of all deductions in IQC, of sentences of $\mathcal{L}[C]$ from finite sets of sentences of $\mathcal{L}[C]$. We need a coding of finite sequences of natural numbers (for example the primitive recursive coding from [74]) and a concatenation operation * so that if $w, v$ code $\left\langle a_{0}, \ldots, a_{n-1}\right\rangle$ and $\left\langle a_{n}, \ldots, a_{n+m-1}\right\rangle$ respectively then $w * v$ is a code for $\left\langle a_{0}, \ldots, a_{n+m-1}\right\rangle$. If $\alpha$ is a function from numbers to numbers then $\bar{\alpha}(n)$ codes $\langle\alpha(0), \ldots, \alpha(n-1)\rangle$. All these ingredients of the proof are effective.

The universal model is based on a spread with spread-law $\Sigma$ and correlation law $\langle G, \Gamma\rangle$ defined step by step so that for each sequence code $w$ :

(i) $\Sigma(w) \in\{0,1\}$, and $w$ is permitted if and only if $\Sigma(w)=0$.

(ii) If $\Sigma(w)=0$ then $G(w) \subseteq C$ and $\Gamma(w)$ is a finite set of sentences of $\mathcal{L}[G(w)]$ and there is at least one $q$ such that $\Sigma(w *\langle q\rangle)=0$.

(iii) If $\Sigma(w *\langle q\rangle)=0$ then one of the following happens:

(a) $G(w) \subsetneq G(w *\langle q\rangle)$ and $\Gamma(w)=\Gamma(w *\langle q\rangle)$,

(b) $G(w)=G(w *\langle q\rangle)$ and $\Gamma(w) \subsetneq \Gamma(w *\langle q\rangle)$.

(c) $G(w)=G(w *\langle q\rangle)$ and $\Gamma(w)=\Gamma(w *\langle q\rangle)$.

The definition begins with $\Sigma(\langle\rangle)=0, \Gamma(\langle\rangle)=\emptyset$ and $G(\langle\rangle)=\left\{c_{(0,0)}, c_{(0,1)}, c_{(0,2)}, \ldots\right\}$, and the construction is arranged so that $\Sigma(w *\langle q\rangle)$ depends on whether the length of $w$ is of the form $3 k, 3 k+1$ or $3 k+2$. In the $3 k$ case $q=0$ is allowed, and (iii)(c) holds, if and only if it is not the case that $\left(\mathrm{A}_{\mathrm{j}_{1}(\mathrm{k})}\right.$ is a sentence of $\mathcal{L}[D(w)]$ and $d_{i}$ proves $\Gamma(w) \vdash \mathrm{A}_{\mathrm{j}_{1}(\mathrm{k})}$ for some $i<3 k$ ); while $q=1$ is allowed if and only if $\mathrm{A}_{\mathrm{j}_{1}(\mathrm{k})}$ is a sentence of $\mathcal{L}[D(w)]$ (in which case $\mathrm{A}_{\mathrm{j}_{1}(\mathrm{k})}$ is put into $\left.\Gamma(w *\langle 1\rangle)\right)$. In the $3 k+1$ case every $q$ is allowed, and the finitely many distinct new constants $c_{(k+1,0)}, \ldots, c_{(k+1, q)}$ from $C$ are put into $G(w *\langle q\rangle)$ so (iii)(a) holds; this ensures the availability of new constants for witnesses. In the $3 k+2$ case we look back to the $3 k$ step and check whether $A_{j_{1}(\mathrm{k})}$ was added to $\Gamma$ then, and if so whether $A_{j_{1}(k)}$ was a disjunction or an existential sentence or neither, and decide accordingly whether to allow only $q=0$ with (iii)(c), or two or infinitely many $q \geq 1$ with (iii)(b) enforcing the disjunction or existence property respectively.

Then the universal modified Kripke model $\mathcal{V}=\{V, \leq, D, \Vdash\}$ is defined by setting $V=\{\alpha: \forall x \Sigma(\bar{\alpha}(x))=0\}$ where

(iv) For each $\alpha \in V: \Gamma_{\alpha}=\bigcup_{x} \Gamma(\bar{\alpha}(x))$ and $G_{\alpha}=\bigcup_{x} G(\bar{\alpha}(x))$ and the domain $D(\alpha)$ is the set of all constants occurring in $\Gamma_{\alpha}$ (so $D(\alpha) \subseteq G_{\alpha}$ ). 
(v) If $\alpha, \beta \in V$ then $\alpha \leq \beta$ if and only if $\Gamma_{\alpha} \subseteq \Gamma_{\beta}$.

(vi) For each $\alpha \in V$ and each prime sentence $\mathrm{P}$ of $\mathcal{L}[D(\alpha)]$ (including $\perp$ ): $\alpha \Vdash \mathrm{P}$ if and only if $\mathrm{P} \in \Gamma_{\alpha}$ or $\perp \in \Gamma_{\alpha}$.

The construction was designed to have the following properties, for each $\alpha \in V$ and each $n$ and $m$ :

(vii) $\Gamma_{\alpha}$ is $D(\alpha)$-saturated.

(viii) If $\mathrm{A}_{n}, \mathrm{~A}_{m}$ are sentences of $\mathcal{L}[D(\alpha)]$ then $\mathrm{A}_{n} \supset \mathrm{A}_{m} \in \Gamma_{\alpha}$ if and only if for each $\beta \geq \alpha$ : if $\mathrm{A}_{n} \in \Gamma_{\beta}$ then also $\mathrm{A}_{m} \in \Gamma_{\beta}$.

(ix) If $\mathrm{A}_{n}$ is a sentence of $\mathcal{L}[D(\alpha)]$ of the form $\forall \mathrm{xB}(\mathrm{x})$, then $\mathrm{A}_{n} \in \Gamma_{\alpha}$ if and only if for all $\beta \geq \alpha: \mathrm{B}(\mathrm{a}) \in \Gamma_{\beta}$ for all $a \in D(\beta)$.

The "only if" directions of (viii) and (ix) are immediate from (vii). Proving the "if" direction of each requires the construction of an appropriate subfan of the spread $\Sigma$. The subfan for (viii) is chosen so that each branch $\beta$ satisfies

$\left(\mathrm{a}^{\prime}\right) \beta(3 j(0, n))=1\left(\right.$ so $\left.\mathrm{A}_{n} \in \Gamma_{\beta}\right)$.

$\left(\mathrm{b}^{\prime}\right) \beta(3 k) \geq \alpha(3 k)$ for all $k$ (so if $\mathrm{A}_{j_{1}(k)} \in \Gamma(\bar{\alpha}(3 k+1))$ then also $\mathrm{A}_{j_{1}(k)} \in \Gamma(\bar{\beta}(3 k+1))$, and so $\left.\Gamma_{\alpha} \subseteq \Gamma_{\beta}\right)$.

$\left(\mathrm{c}^{\prime}\right) \beta(1)=\max (\alpha(1), m, n)+2$, and $\beta(3 k+1)=\max (\alpha(3 k+1), \beta(3 k-2), m, n)+2$ for all $k \geq 1$ (so $G(\bar{\beta}(3 k+2))$ always contains two fresh constants).

$\left(\mathrm{d}^{\prime}\right) \beta(3 k+2)=\alpha(3 k+2)$ except in the case that $\alpha(3 k)=0$ and $\beta(3 k)=1$ and $\mathrm{A}_{j_{1}(k)}$ is a disjunctive or existential sentence; then $\beta(3 k+2) \in\{1,2\}$ (if $\mathrm{A}_{j_{1}(k)}$ is a disjunction) or $\beta(3 k+2) \in\{c, d\}$ where $c, d$ are the two fresh constants (if $\mathrm{A}_{j_{1}(k)}$ is existential).

Now suppose $\mathrm{A}_{n}, \mathrm{~A}_{m}$ are such that for each $\beta \in V$ with $\alpha \leq \beta$ : If $\mathrm{A}_{n} \in \Gamma_{\beta}$ then $\mathrm{A}_{m} \in \Gamma_{\beta}$. Then by $\left(\mathrm{a}^{\prime}\right)$ and $\left(\mathrm{b}^{\prime}\right)$ with the Fan Theorem there is an $x_{0}$ such that $\mathrm{A}_{m} \in \Gamma\left(\bar{\beta}\left(x_{0}\right)\right)$ for every $\beta$ in the subfan. By fan induction using $\left(b^{\prime}\right)$ and $\left(\mathrm{d}^{\prime}\right)$ : if $\beta$ is in the subfan and $x \leq x_{0}$ then $\Gamma(\bar{\beta}(x)), \Gamma_{\alpha}, \mathrm{A}_{n} \vdash \mathrm{A}_{m}$. Taking $x=0$ and using the Deduction Theorem, finally $\Gamma_{\alpha} \vdash \mathrm{A}_{n} \supset \mathrm{A}_{m}$.

The proof of (ix) is similar. It follows that $\Gamma_{\alpha}$ consists exactly of those sentences of $\mathcal{L}[D(\alpha)]$ which are forced at $\alpha$, and this is enough (together with the construction of $\Gamma$ ) to establish Veldman's completeness theorem. Informal Markov's Principle is not needed in the proof.

\section{Acknowledgements}

I am grateful to the students of the Graduate Program in Logic and Algorithms (MП $\Lambda$ A) in Athens, especially to Garyfallia Vafeiadou and Nikos Vaporis, for reviving and expanding my enthusiasm for this subject over the past few years. Anne Troelstra and Jaap van Oosten unwittingly showed me by example how an encyclopedia article should be written. Mark van Atten, Garyfallia Vafeiadou, Richard Vesley, Richard Zach and Yiannis Moschovakis generously read and commented on an earlier version of the manuscript. I also thank Marcel Guillaume and Paolo Mancosu for providing helpful information. All remaining errors and omissions are exclusively mine. 


\section{References}

[1] P. Aczel [1968], Saturated intuitionistic theories, in H. Schmidt, K. Schütte and H.J. Thiele, eds., [1968], Contributions to Mathematical Logic, North-Holland, Amsterdam.

[2] J. Avigad and S. Feferman [1998], Gödel's functional ("Dialectica") interpretation, Ch. V of [31], 337-405.

[3] E. W. Beth [1956], Semantic construction of intuitionistic logic, Kon. Ned. Akad. v. Wet. Afd. Let. Med. Nieuwe Serie 19/11, 357-388.

[4] E. W. Beth [1959], The Foundations of Mathematics, North-Holland, Amsterdam.

[5] M. Barzin and A. Errera [1927], Sur la logique de M. Brouwer, Bull. Acad. Royale Belgique 13, 56-71.

[6] M. Beeson [1985], Foundations of Constructive Mathematics, Springer Verlag, Berlin.

[7] P. Bernays [1926], Axiomatische Untersuchung des Aussagenkalküls der 'Principia Mathematica', Math. Zeitschrift 25, 305.

[8] E. Bishop [1967], Foundations of Constructive Analysis, McGraw-Hill, New York.

[9] D. Bridges and F. Richman [1987], Varieties of Constructive Mathematics, Cambridge Univ. Press, Cambridge.

[10] L. E. J. Brouwer [1907], Over de Grondslagen der Wissenschafte, Thesis, Amsterdam. English trans. On the foundations of mathematics in Heyting, ed., Brouwer [1975, 11$101]$.

[11] L. E. J. Brouwer [1908], De onbetrouwbaarheid der logische principes, Tijdschrift voor Wijsbegeerte 2, 152-158. English trans. The unreliability of the logical principles in Heyting, ed., Brouwer [1975, 107-111].

[12] L. E. J. Brouwer [1909], Het wezen der meetkunde, Amsterdam. English trans. The nature of Geometry in Heyting, ed., Brouwer [1975, 112-120].

[13] L. E. J. Brouwer [1912], Intuitionism and formalism, English trans. by A. Dresden, Bull. Amer. Math. Soc. 20, 81-96. Reprinted in Benacerraf and Putnam [1983, 77-89].

[14] L. E. J. Brouwer [1918], Begrundung der Mengenlehre unabhangig vom logischen Satz vom ausgeschlossenen Dritten. Erster Teil: Allgemeine Mengenlehre, Ver. Kon. Akad. v. Wet. I, 12, no. 5. Reprinted in Heyting, ed., Brouwer [1975, 150-190].

[15] L. E. J. Brouwer [1919], Begrundung der Mengenlehre unabhangig vom logischen Satz vom ausgeschlossenen Dritten. Zweiter Teil: Theorie der Punktmengen, Ver. Kon. Akad. v. Wet. I, 12, no. 7. Reprinted in Heyting, ed., Brouwer [1975, 191-221]. 
[16] L. E. J. Brouwer [1923], Intuitionistische splitsing van mathematische grondbegrippen, Ver. Kon. Akad. v. Wet. 32, 877-880. English trans. by W. P. van Stigt in Mancosu [1998, 286-289].

[17] L. E. J. Brouwer [1923a], Über die Bedeutung des Satzes vom ausgeschlossen Dritten in der Mathematik, insbesondere in der Funktionentheorie, Journal für die reine und angewandte Mathematik 154, 1-7. English trans. by S. Bauer-Mengelberg in van Heijenoort [1967, 334-341]. Cf. Brouwer [1954].

[18] L. E. J. Brouwer [1923b], Begründung der Funktionlehre unabhängig vom Logischen Satz der ausgeschlossen Drutten, Ver. Akad. Wet. Amsterdam 13 No. 2.

[19] L. E. J. Brouwer [1924], Beweis, dass jede volle Funktion gleichmässig stetig ist, Kon. Ned. Akad. v. Wet. Proc. 27, 189-193. Reprinted in Heyting, ed., Brouwer [1975, 286-290].

[20] L. E. J. Brouwer [1924a], Intuitionistische Zerlegung mathematischer Grundbegriffe, Jber. Deutsch Math. Verein. 33, 251-256. Reprinted in Heyting, ed., Brouwer [1975, 275-280]. English trans. by W. P. van Stigt in Mancosu [1998, 290-292].

[21] L. E. J. Brouwer [1925], Zur Begründung der intuitionistische Mathematik I, Math. Annalen 93, 244-257.

[22] L. E. J. Brouwer [1927], Über Definitionsbereiche von Funktionen, Math. Annalen 97, 60-75. English trans. of $\S 1-3$ : On the domains of definition of functions by S. Bauer-Mengelberg in van Heijenoort [1967, 446-463].

[23] L. E. J. Brouwer [1927a], Zur intuitionistische Zerlegung mathematischer Grundbegriffe, Jber. Deutsch Math. Verein. 36, 127-129. Reprinted in Heyting, ed., Brouwer [1975, 295-297]. English trans. by W. P. van Stigt in Mancosu [1998, 293-295].

[24] L. E. J. Brouwer [1928], Intuitionistische Betrachtungen über den Formalismus, KNAW Proc. 31, 374-379. Also Sitzber. Preuss. Akad. Wiss. (1928), 48-52. English trans. of $\S$ I by S. Bauer-Mengelberg in van Heijenoort [1967, 490-492].

[25] L. E. J. Brouwer [1930], Die Struktur des Kontinuums, in Heyting, ed., Brouwer [1975, 429-440].

[26] L. E. J. Brouwer [1952], Historical background, principles and methods of intuitionism, South-African Jour. Sci. 49, 139-146.

[27] L. E. J. Brouwer [1954], Addenda and corrigenda and Further addenda and corrigenda to Brouwer [1923a], English trans. by S. Bauer-Mengelberg, C. Berndes Franck, D. van Dalen and the editor in van Heijenoort [1967, 341-345].

[28] L. E. J. Brouwer [1954a], Points and spaces, Canad. Jour. Math. 6, 1-17. Reprinted in Heyting, ed., Brouwer [1975, 522-538].

[29] L. E. J. Brouwer [1975], Collected Works, 1, edited by A. Heyting, North-Holland, Amsterdam.

[30] L. E. J. Brouwer [1981], Brouwer's Cambridge Lectures on Intuitionism, edited by D. van Dalen, Cambridge Univ. Press, Cambridge. 
[31] S. Buss, ed. [1998], The Handbook of Proof Theory, Elsevier, Amsterdam and New York.

[32] A. Church [1928], On the law of excluded middle, Bull. Amer. Math. Soc. 34, 75-78.

[33] A. Church [1936], An unsolvable problem of elementary number theory, Amer. Jour. Math. 58, 345-363.

[34] A. Church [1936a], A note on the Entscheidungsproblem, Jour. Symb. Logic 1, 40-41. Correction, Ibid., 101-102.

[35] R. Dedekind [1888], Was sind und was sollen die Zahlen, Braunschweig. English trans. by W. Beman in R. Dedekind [1901], Essays on the Theory of Numbers, Open Court, Chicago, reprinted by Dover (1963), 31-105.

[36] A. G. Dragalin [1988] Mathematical Intuitionism: Introduction to Proof Theory, Trans. Math. Mon. 67, Amer. Math. Soc., Providence, R.I. English trans. by E. Mendelsohn of the 1979 Russian version.

[37] M. Dummett [1977] Elements of Intuitionism, Clarendon Press, Oxford.

[38] V. Dyson and G. Kreisel [1961], Analysis of Beth's semantic construction of intuitionistic logic, Stanford Report.

[39] W. Dzik [2004], Chains of structurally complete predicate logics with the application of Prucnal's substitution, Reports on Math. Logic 38, 37-48.

[40] H. Friedman [1975], The disjunction property implies the existence property, Proc. Nat. Acad. Sci. 72, 2877-2878.

[41] D. Gabbay and D. H. J. de Jongh [1974], A sequence of finitely axiomatizable intermediate logics with the disjunction property, Jour. Symb. Logic 39, 67-78.

[42] G. Gentzen [1933], Über das Verhältnis zwischen intuitionistischer und klassischer Logik, accepted by Math. Annalen (1933) but withdrawn; published in Arch. Math. Logik Grund. 16 (1974), 119-132. English trans. in Szabo, ed., Gentzen [1967, 53-67].

[43] G. Gentzen [1935], Untersuchungen über das logische Schliessen, Math. Zeitschrift 39 (1934-5), 176-210, 405-431.

[44] G. Gentzen [1967], Gentzen: Collected Papers, edited by M. E. Szabo, NorthHolland, Amsterdam.

[45] S. Ghilardi [1999], Unification in intuitionistic logic, Jour. Symb. Logic 64, 859-880.

[46] V. Glivenko [1928], Sur la logique de M. Brouwer, Bull. Acad. Royale de Belgique 14, 225-228.

[47] V. Glivenko [1929], Sur quelques points de la logique de M. Brouwer, Bull. Acad. Royale de Belgique 15, 183-188. English trans. by A. Rocha in Mancosu [1998, 301$305]$.

[48] K. Gödel [1932], Über Vollstandigkeit und Widerspruchfreiheit, Ergebnisse eines math. Koll. 3 (for 1930-1931, pub. 1932), 12-13. 
[49] K. Gödel [1932a], Zum intuistischen Aussagenkalkül, Akad. Wiss. Wien, Math.naturwiss. Klasse, Anzeiger 69, 65-66.

[50] K. Gödel [1933], Über Unabhängigkeitsbeweise im Aussagenkalkül, Ergebnisse eines math. Koll. 4 (for 1932, pub. 1933), 9-10.

[51] K. Gödel [1933a], Zur intuitionistischen Arithmetik und Zahlentheorie, Ergebnisse eines math. Koll., 4 (for 1932, pub. 1933), 34-38.

[52] K. Gödel [1958], Über eine bisher noch nicht benützte Erweiterung des finiten Standpunktes, Dialectica 12, 280-287.

[53] R. Harrop [1956], On disjunctions and existential statements in intuitionistic systems of logic, Math. Annalen 132, 347-361.

[54] D. Hesseling [2003], Gnomes in the Fog: The Reception of Brouwer's Intuitionism in the 1920s, Birkhaüser, Basel.

[55] A. Heyting [1930], Sur la logique intuitionniste, Bull. Acad. Royale Belgique 16, 957963. English trans. by A. Rocha in Mancosu [1998, 306-310].

[56] A. Heyting [1930I], Die formalen Regeln der intuitionistischen Logik, Sitzber. Preuss. Akad. Wiss. (phys.-math. Klasse), Berlin, 42-56. English trans. in Mancosu [1998, 311-327].

[57] A. Heyting [1930II], Die formalen Regeln der intuitionistischen Mathematik II, Sitzber. Preuss. Akad. Wiss. (phys.-math. Klasse), Berlin, 57-71.

[58] A. Heyting [1930III], Die formalen Regeln der intuitionistischen Mathematik III, Sitzber. Preuss. Akad. Wiss. (phys.-math. Klasse), Berlin, 158-169.

[59] A. Heyting [1946], On weakened quantification, Jour. Symb. Logic 11, 119-121.

[60] A. Heyting [1956], Intuitionism: An Introduction, North-Holland, Amsterdam.

[61] A. Heyting [1961], Infinitistic methods from a finitist point of view, in Infinitistic Methods, Proceedings of the Symposium on Foundations of Mathematics (Warsaw 1959), Pergamon Press, 185-192.

[62] D. Hilbert and W. Ackermann [1928], Grundzüge der theoretischen Logik, Springer Verlag, Berlin.

[63] D. Hilbert and P. Bernays [1934], Grundlagen der Mathematik 1, Springer Verlag, Berlin.

[64] D. Hilbert and P. Bernays [1939], Grundlagen der Mathematik 2, Springer Verlag, Berlin.

[65] R. Iemhoff [2001], On the admissible rules of intuitionistic propositional logic, Jour. Symb. Logic 66, 281-294.

[66] R. Iemhoff [2005], Intermediate logics and Visser's rules, Notre Dame Jour. Form. Logic 46, 65-81. 
[67] D. H. J. de Jongh [1970], The maximality of the intuitionistic propositional calculus with respect to Heying's arithmetic, Jour. Symb. Logic 36, 606.

[68] S. C. Kleene [1945], On the interpretation of intuitionistic number theory, Jour. Symb. Logic 10, 109-124.

[69] S. C. Kleene [1952], Introduction to Metamathematics, North-Holland, Amsterdam.

[70] S. C. Kleene [1962], Disjunction and implication under implication in elementary intuitionistic formalisms, Jour. Symb. Logic 27, 11-18. Addendum (1963) in Jour. Symb. Logic 28, 154-156.

[71] S. C. Kleene [1965], Classical extensions of intuitionistic mathematics, in Y. BarHillel, ed., Logic, Methodology and Philosophy of Science, North-Holland, Amsterdam, 31-44.

[72] S. C. Kleene [1967], Constructive functions in "The Foundations of Intuitionistic Mathematics", in Logic, Methodology and Philos. of Science III, NorthHolland, Amsterdam, 137-144.

[73] S. C. Kleene [1969], Formalized recursive functionals and formalized realizability, Mem. Amer. Math. Soc. 89

[74] S. C. Kleene and R. E. Vesley [1965], The Foundations of Intuitionistic Mathematics, Especially in Relation to Recursive Functions, North-Holland, Amsterdam.

[75] A. Kolmogorov [1925], O principe tertium non datur, Matematiceskij Sbornik 32, 646-667. English trans. in van Heijenoort [1967, 414-437].

[76] A. Kolmogorov [1932], Zur Deutung der intuitionistische Logik, Math. Zeitschrift 35, 58-65. English trans. in Mancosu [1998, 328-334].

[77] P. Krauss [1992], A constructive interpretation of classical mathematics, Math. Schriften Kassel, Preprint No. 5/92, 1992.

[78] G. Kreisel [1958], Mathematical significance of consistency proofs, Jour. Symb. Logic 23, 155-181.

[79] G. Kreisel [1959], The non-derivability of $\neg \forall x A(x) \rightarrow \exists x \neg A(x)$, A primitive recursive, in intuitionistic formal systems (abstract), Jour. Symb. Logic 23 (No. 4 for 1958, publ. 1959), 456-457.

[80] G. Kreisel [1962], On weak completeness of intuitionistic predicate logic, Jour. Symb. Logic 27, 139-158.

[81] G. Kreisel [1968], Lawless sequences of natural numbers, Comp. Math. 20, 222-248.

[82] G. Kreisel and A. S. Troelstra [1970], Formal systems for some branches of intuitionistic analysis, Ann. Math. Logic 1, 229-387.

[83] S. Kripke [1965], Semantical analysis of intuitionistic logic, in Formal Systems and Recursive Functions (J. Crossley and M. Dummett, eds.), North-Holland, Amsterdam, 92-130. 
[84] M. D. Krol' [1973], A topological model for intuitionistic analysis with Kripke's Schema, Zeitsch. math. Logik Grund. 24, 427-436.

[85] I. Loeb [2005], Equivalents of the (Weak) Fan Theorem, Ann. Pure and Appl. Logic 132, 51-66.

[86] P. Mancosu [1998], From Brouwer to Hilbert: The Debate on the Foundations of Mathematics in the 1920s, Oxford Univ. Press, Oxford and New York.

[87] P. Mancosu and W. van Stigt [1998], Intuitionistic logic, in Mancosu [1998, 275-285].

[88] P. Mancosu, R. Zach and C. Badesa [200?], The development of mathematical logic from Russell to Tarski, in L. Haaparanta, ed., The Development of Modern Logic: A Philosophical and Historical Handbook, Oxford University Press, Oxford.

[89] P. Martin-Löf [1984] Intuitionistic Type Theory, Bibliopolis, Naples.

[90] G. Mints [1976], Derivability of admissible rules, Jour. Soviet Math. 6 (1976), 417-421.

[91] J. R. Moschovakis [1971], Can there be no non-recursive functions?, Jour. Symb. Logic 36, 309-315.

[92] J. R. Moschovakis [1996], A classical view of the intuitionistic continuum, Ann. Pure and Appl. Logic 81, 9-24.

[93] J. R. Moschovakis [2003], Classical and constructive hierarchies in extended intuitionistic mathematics, Jour. Symb. Logic 68, 1015-1043.

[94] J. R. Moschovakis [2004], Intuitionistic Logic, in the Stanford On-Line Encyclopedia of Philosophy, http : //www.plato.stanford.edu.

[95] A. Mostowski [1948], Proofs of non-deducibility in intuitionistic functional calculus, Jour. Symb. Logic 13, 204-207.

[96] J. Myhill, A. Kino and R. Vesley, eds. [1970], Intuitionism and Proof Theory, North-Holland, Amsterdam.

[97] D. Nelson [1947], Recursive functions and intuitionistic number theory, Trans. Amer. Math. Soc. 61, 307-368.

[98] M. Presburger [1930], Über die Vollstandigkeit eines gewissen Systems der Arithmetik ganzer Zahlen in Welchem die Addition als einzige Operation hervortritt, Sprawozdanie z I Kongresu Matematyków Krajów Slowiańskich (Comptes-rendus du I Congrés des Mathématiciens des Pays Slaves), Warszawa 1929, Warsaw 92-101, 395.

[99] H. Rasiowa [1955], Algebraic models of axiomatic theories, Fund. Math. 41, 291-310.

[100] H. Rasiowa and R. Sikorski [1954], On existential theorems in non-classical functional calculi, Fund. Math. 41, 21-28.

[101] H. Rasiowa and R. Sikorski [1963], The Mathematics of Metamathematics, Warsaw. 
[102] J. B. Rosser [1936], Extensions of some theorems of Gödel and Church, Jour. Symb. Logic 1, 87-91.

[103] V. Rybakov [1997], Admissibility of Logical Inference Rules, Elsevier, Amsterdam.

[104] H. Schütte [1950], Schlussweisen-Kalküle der Prädikatenlogik, Math. Annalen 122, 47-65.

[105] D. Scott [1968], Extending the topological interpretation to intuitionistic analysis, Comp. Math. 20, 194-210.

[106] D. Scott [1970], Extending the topological interpretation to intuitionistic analysis II, in [96], 235-255.

[107] D. Scott [1979], Identity and existence in intuitionistic logic, in Applications of Sheaves (M. Fourman, C. Mulvey and D. Scott, eds.), Springer Verlag, Berlin, 660696.

[108] C. Smorynski [1973], Applications of Kripke models, Ch. V of Troelstra, ed. [112], 324-391.

[109] C. Spector [1962], Provably recursive functionals of analysis: a consistency proof of analysis by an extension of principles formulated in current intuitionistic mathemat$i c s$, in J. C. E. Dekker, ed., Recursive Function Theory, AMS Proc. Symp. Pure Math V., Providence, 1-27.

[110] A. S. Troelstra [1968], The scientific work of A. Heyting, Comp. Math. 20, 3-12.

[111] A. S. Troelstra [1971], Notions of realizability for intuitionistic arithmetic and intuitionistic arithmetic in all finite types, in J. Fenstad, ed. [1971], The Second Scandinavian Logic Symposium, North-Holland, Amsterdam, 369-405.

[112] A. S. Troelstra, ed. [1973], Metamathematical Investigations of Intuitionistic Arithmetic and Analysis, Springer Lecture Notes in Math. 344, Springer Verlag, Berlin.

[113] A. S. Troelstra [1973a], Notes on intuitionistic second order arithmetic, in A. Mathias and H. Rogers, eds. [1973], Cambridge Summer School in Mathematical Logic, Springer, Berlin, 171-205.

[114] A. S. Troelstra [1974], Note on the fan theorem, Jour. Symb. Logic 39, 584-596.

[115] A. S. Troelstra [1977], Choice Sequences, a Chapter of Intuitionistic Mathematics, Clarendon Press, Oxford.

[116] A. S. Troelstra [1991], History of constructivism in the twentieth century, ITLI (Inst. v. Taal, Logica en Informatie) Prepublication Series ML-91-05, Amsterdam.

[117] A. S. Troelstra [1998], Realizability, Ch. VI of [31], 407-473.

[118] A. S. Troelstra and D. van Dalen [1988], Constructivism in Mathematics: An Introduction, 1 and 2, North-Holland, Amsterdam. 
[119] M. van Atten [2005], Luitzen Egbertus Jan Brouwer, in the Stanford On-Line Encyclopedia of Philosophy, http : //www.plato.stanford.edu.

[120] M. van Atten and J. Kennedy [2007?], Gödel's Logic, in this volume.

[121] D. van Dalen [1984], How to glue analysis models, Jour. Symb. Logic 49, 1339-1349.

[122] D. van Dalen [1990], The war of the frogs and the mice, or the crisis of the Mathematische Annalen, Math. Intelligencer 12(4), 17-31.

[123] D. van Dantzig [1947], On the principles of intuitionistic and affirmative mathematics, Ver. Kon. Akad. v. Wet. 50, 918-929, 1092-1103; also Indagationes math. 9, 429-440, 506-517.

[124] J. van Heijenoort [1967], From Frege to Gödel: A Source Book in Mathematical Logic 1897-1931, Harvard Univ. Press, Cambridge, Mass.

[125] J. van Oosten [1991], A semantical proof of de Jongh's theorem, Arch. Math. Logic 31, 105-114.

[126] J. van Oosten [2002], Realizability: a historical essay, Math. Struct. Comp. Sci. 12, 239-263.

[127] W. P. van Stigt [1990], Brouwer's Intuitionism, North-Holland, Amsterdam.

[128] W. Veldman [1976], An intuitionistic completeness theorem for intuitionistic predicate logic, Jour. Symb. Logic 41(1), 159-166.

[129] R. E. Vesley [1970], A palatable alternative to Kripke's Schema, in [96].

[130] R. E. Vesley [1980], Intuitionistic analysis: The search for axiomatization and understanding, in The Kleene Symposium, North- Holland, Amsterdam, 317-331.

[131] A. Visser [1999], Rules and arithmetics, Notre Dame Jour. Form. Logic 40, 116-140.

[132] J. von Plato [2007?], Gentzen's Logic, in this volume.

[133] R. Wavre [1926], Logique formelle et logique empiriste, Revue de Métaphysique et de Morale 33, 65-75.

[134] R. Zach [1999], Completeness before Post, Bull. Symb. Logic 5(3), 303-330. 